Revue musicale OICRM

\title{
Le paradoxe du clavicorde et la technique de Bach au clavier
}

\section{Christophe d'Alessandro}

Volume 6, numéro 1, 2019

Documenter, analyser et révéler la création sonore en interprétation musicale

URI : https://id.erudit.org/iderudit/1062430ar

DOI : https://doi.org/10.7202/1062430ar

Aller au sommaire du numéro

\section{Éditeur(s)}

Observatoire interdisciplinaire de création et recherche en musique (OICRM)

ISSN

2368-7061 (numérique)

Découvrir la revue

Citer cet article

d'Alessandro, C. (2019). Le paradoxe du clavicorde et la technique de Bach au clavier. Revue musicale OICRM, 6(1), 87-112. https://doi.org/10.7202/1062430ar

\section{Résumé de l'article}

Le jeu du clavicorde demande un contrôle paradoxal : il faut abaisser la touche suffisamment vite pour produire un son suffisamment fort, mais aussi veiller à garder la justesse d'intonation, c'est-à-dire ne pas trop déplacer la corde. Les sources historiques sur le jeu du clavicorde dans le cercle de Jean-Sébastien Bach décrivent une technique de clavier ( tire " ou " Schnellen ") qui semble particulièrement adaptée au contrôle simultané de la vitesse et de la profondeur du toucher. Après une étude théorique qui établit les rapports entre mouvement de la touche, intensité sonore et intonation, une étude expérimentale mesure ces quantités pour un toucher " poussé », vertical et un toucher « tiré ", avec retrait du bout du doigt vers l'intérieur de la main. Les résultats indiquent que le contrôle paradoxal demandé par la mécanique spécifique du clavicorde est avantageusement réalisé par le geste décrit dans les sources historiques (un mouvement de retrait circulaire du bout du doigt). 


\title{
Le paradoxe du clavicorde et la technique de Bach au clavier
}

\author{
Christophe d'Alessandro
}

\begin{abstract}
Résumé
Le jeu du clavicorde demande un contrôle paradoxal : il faut abaisser la touche suffisamment vite pour produire un son suffisamment fort, mais aussi veiller à garder la justesse d'intonation, c'est-à-dire ne pas trop déplacer la corde. Les sources historiques sur le jeu du clavicorde dans le cercle de Jean-Sébastien Bach décrivent une technique de clavier (" tire " ou "Schnellen ") qui semble particulièrement adaptée au contrôle simultané de la vitesse et de la profondeur du toucher. Après une étude théorique qui établit les rapports entre mouvement de la touche, intensité sonore et intonation, une étude expérimentale mesure ces quantités pour un toucher "poussé ", vertical et un toucher " tiré », avec retrait du bout du doigt vers l'intérieur de la main. Les résultats indiquent que le contrôle paradoxal demandé par la mécanique spécifique du clavicorde est avantageusement réalisé par le geste décrit dans les sources historiques (un mouvement de retrait circulaire du bout du doigt).

Mots clés : acoustique ; Jean-Sébastien Bach ; clavicorde ; jeu instrumental ; technique claviéristique; toucher.
\end{abstract}

\begin{abstract}
Playing the clavichord demands a paradoxical control: one should press the key fast enough, but without pushing the string up, in order to produce a sufficiently loud sound but also to maintain a correct intonation. Historical sources on keyboard technique in Johann Sebastian Bach's circle describe finger motions ("tire" or "Schnellen") that seem particularly suited to the simultaneous control of the speed and depth of touch. After a theoretical study that establishes the relationship between the movement of the key, sound intensity and intonation, an experimental study measures these quantities for a "pushed" or vertical touch and for a "pulled" touch, with a gliding motion of the fingertip towards the hand palm. The results indicate that the paradoxical control demanded by the specific mechanics of the clavichord is advantageously achieved by the gesture described in historical sources (a circular withdrawal movement of the fingertip).
\end{abstract}

Keywords: acoustics; Johann Sebastian Bach; clavichord; keyboard technique; performance; touch. 
LE PARADOXE DU CLAVICORDE

La recherche d'une pratique historiquement située des instruments à clavier puise traditionnellement à plusieurs sources : les partitions, les traités pour l'apprentissage de l'instrument, les descriptions de concerts d'époque, l'iconographie musicale, et bien sûr l'organologie. En effet, les gestes les plus appropriés pour manœuvrer au mieux un instrument donné, ici le clavicorde, sont au moins en partie induits par ses propriétés statiques et dynamiques.

Le clavicorde est apparu au Bas Moyen Âge en Europe ${ }^{1}$. Son intensité sonore est plutôt faible, ce qui permet une utilisation intensive et discrète. Le son délicat du clavicorde invite à une écoute très attentive et concentrée. Mais surtout, le contrôle du mouvement de la touche doit être particulièrement précis ${ }^{2}$. Le mécanisme très simple du clavicorde, le doigt restant en contact avec la corde après la production du son par l'intermédiaire du levier de la touche et de la tangente, implique que la moindre variation d'enfoncement ou de vitesse change le timbre, l'intensité, la durée du son. La corde sonne tant que corde et tangente restent en contact.

Dans le présent essai, nous tentons le rapprochement entre la technique du clavier associée à l'école de Jean-Sébastien Bach (et de ses fils Carl Philipp Emanuel et Wilhelm Friedemann) et les propriétés mécaniques et acoustiques spécifiques du clavicorde. Les musiciens de ce cercle pratiquent toutes les sortes d'instruments à clavier, à la fois l'orgue, le clavecin, le pianoforte, et bien sûr le clavicorde. Le clavicorde est systématiquement mentionné comme l'instrument $^{3}$ pédagogique par excellence pour former le jeu ${ }^{4}$. Un exemple parmi beaucoup d'autres, en 1732, J.G. Walther, cousin et ami de Bach, note à l'article "Clavicordo » de son lexique musical ${ }^{5}$ : «Cet instrument très commun est pour ainsi dire la première grammaire de tous les joueurs [d'instruments à clavier], car s'ils le maîtrisent, ils joueront également bien l'épinette, le clavecin, la régale, le positif et le grand orgue ${ }^{6}$ ». L'hypothèse défendue ici est que cette qualité pédagogique reconnue au clavicorde est spécifique-

\footnotetext{
1 La première représentation connue, datée de 1425, figure sur un bas relief de bois sculpté qui provient de la cathédrale de Minden, en Allemagne. Une étude organologique, musicologique et iconographique d'ensemble sur le clavicorde se trouve dans l'ouvrage de référence de Brauchli (1998).

$2 \quad$ Il est intéressant de consulter les points de vue de facteurs de clavicorde, rapportés par exemple par Tournay 2009 et Jobin 1992.

3 Le clavicorde est souvent dénommé tout simplement « clavier » à l'époque en Allemagne, car c'est l'instrument à clefs (c'est-à-dire à touches) par excellence.

4 Le clavicorde comme instrument pédagogique est discuté par exemple dans Baillot 1992, Adlam 1998, Brauchli 2002, Speestra 2004 et Crowell 2004.

$5 \quad$ Walther 1732, p. 169.

6 "Dieses sehr bekannte Instrument, ist, so zu reden, aller Spieler erste Grammatica; denn, so sie dieses mächtig sing, sie auch auf Spinnetten, Clavycymbeln, Regalen, Positiven und Orgeln, zurechte kommen " (traduction de l'auteur, comme toutes les traductions de l'article, sauf indication dans la référence bibliographique. L'orthographe est modernisée comme pour les textes anciens de cet article).
} 
ment liée au mécanisme et aux propriétés acoustiques de l'instrument, qui favorisent à la fois l'écoute et l'éducation somatique du toucher ${ }^{7}$.

L'étude de la dynamique du clavicorde ${ }^{8}$ a montré une relation linéaire entre l'intensité sonore (Sound Pressure Level ou SPL) et la vitesse de la tangente au moment de l'impact avec la corde $(V p)$. Les mesures montrent également que l'accroissement de $V p$ s'accompagne d'un accroissement de fréquence fondamentale $(F 0)$. En effet, un changement de vitesse d'impact entraîne un changement d'enfoncement de la touche, donc un changement de tension de la corde et de hauteur tonale.

En raison du contact maintenu entre la corde et la tangente, donc avec le doigt, le jeu du clavicorde demande un geste paradoxal : pour obtenir à la fois une grande gamme dynamique et une intonation juste, il faut un mouvement du doigt plus ou moins rapide et en même temps une profondeur d'enfoncement de la touche toujours contrôlée. Le paradoxe du clavicorde s'énonce ainsi : à l'instant du contact tangentecorde la tangente doit avoir de la vitesse, mais ne doit pas hausser la corde. Il faudrait donc, à la limite, transmettre toute la quantité de mouvement de la tangente à la corde (quantité de mouvement qui serait entièrement transférée en vibration de la corde) sans la hausser ${ }^{9}$, ni perdre le contact $^{10}$. Dans la pratique, ce type de mouvement paradoxal est impossible à réaliser exactement, mais des compromis entre vitesse d'impact de la tangente et déplacement de la corde sont possibles, en particulier par un contrôle rapide des nuances d'enfoncement dans les tous premiers instants de la production du son.

Comme pour d'autres tâches d'éducation somatique, le problème posé par une contrainte paradoxale se révèle un puissant moyen pour parvenir à un geste plus efficace que le geste d'abaissement vertical spontanément effectué. Nous défendons ici l'idée que c'est la contrainte paradoxale d'un mouvement rapide et en même temps d'un faible déplacement de la touche qui est au cœur de la maîtrise de l'instrument. Nous souhaitons démontrer que les gestes spécifiques au clavicorde, décrits dans des sources historiques, répondent à cette contrainte paradoxale.

La première partie de cet article est une étude du geste au clavicorde, tel qu'il est décrit de façon précise et répétitive à partir du cercle de Jean-Sébastien Bach dans la seconde moitié du XVIII ${ }^{\mathrm{e}}$ siècle. La seconde partie établit les relations entre le mouvement du clavier, la vitesse des tangentes, l'intonation et l'intensité sonore du clavicorde. La dépendance entre force et intonation est établie en théorie. En troisième partie, des mesures sont effectuées en utilisant une technique de jeu

7 "Éducation somatique » est pris ici dans le sens de "méthode qui vise à augmenter l'efficacité et l'aisance des mouvements par le développement de la conscience corporelle (soma)».

8 Voir Thwaites et Fletcher 1981, Alessandro et al. 2006, 2008, Alessandro 2010a.

9 C'est le type de mouvement que l'on observe dans le dispositif connu sous le nom de pendule de Newton : les billes du centre restent immobiles, mais la quantité de mouvement se transmet aux extrémités.

10 Si la pression du doigt se relâche trop, il y a le risque d'un contact intermittent entre corde et tangente, une corde qui « frise » contre la tangente en produisant un bruit inacceptable. Précisons que l'oscillation de la corde sur un clavicorde est principalement dans le plan vertical, bien que les oscillations dans le plan horizontal soient également significatives. 
du clavier dérivée des informations de la première partie et la technique ordinaire avec un mouvement vertical du doigt. Une conclusion sur les vertus pédagogiques du clavicorde est proposée en dernière partie.

\section{À L’ÉCOLE DE JEAN-SÉBASTIEN BACH}

\section{L'expressivité du toucher}

La pratique de l'instrument permet de se rendre compte immédiatement du paradoxe du clavicorde : si l'on joue fort, on a tendance à enfoncer davantage la touche et à hausser le ton. Pour jouer fort et juste, il faut donc contrôler simultanément la vitesse d'attaque et la profondeur d'enfoncement de la touche.

La liaison constamment maintenue entre la corde et le doigt ainsi que l'enfoncement peu profond du clavier ${ }^{11}$ sont des difficultés spécifiques de l'instrument, qui font en même temps sa richesse. Une façon de limiter l'élévation de la tangente, et par conséquent le défaut de justesse, consiste à augmenter la dureté du toucher (voir la formule (2) plus loin). Dans certains instruments, un étouffoir rigide (stoppeur) est disposé à gauche des tangentes afin de limiter le soulèvement de la corde et d'éviter le défaut de justesse tonale. Le toucher devient plus ferme, l'instrument plus difficile à jouer, moins souple et moins agréable sous les doigts. Cette sensation de fermeté est due à l'enfoncement de la touche qui se trouve limité par le stoppeur, lequel oppose alors une résistance fixe un fois que la touche a atteint sa course maximale d'enfoncement. La corde ne risque plus d'être soulevée de manière excessive, la hauteur tonale reste acceptable, quelle que soit la modalité de jeu. Ce n'est pas forcément une bonne solution, ni une solution généralement adoptée dans les instruments historiques.

Plutôt que de durcir le toucher, il est possible de développer des gestes qui autorisent une certaine marge dans les variations entre vitesse et déplacement de la touche au moment du contact avec la corde. On peut utiliser ces gestes pour minimiser le déplacement, en maximisant la vitesse, tout en maintenant le contact corde/tangente.

Toute l'évolution du geste pendant qu'une note sonne a de l'importance. Toute nuance de pression du doigt sur la corde participera à la vie du son. Les mécanismes des autres instruments à clavier (orgue, clavecin, piano, etc.) ne demandent pas un tel contrôle : l'intonation n'est pas affectée par la vitesse du jeu ou l'enfoncement de la touche, sauf dans des cas limites (comme une ouverture incomplète des soupapes d'orgue, ou un impact excessivement fort pour les instruments à marteaux). Le mécanisme de ces instruments (séparation de la noix du marteau du pilote ou échappement de la mécanique au piano, ouverture de la soupape de l'orgue, échappement du bec au clavecin, etc.) est tel que le doigt n'a plus d'effet sur le son tant que la touche est abaissée, bien que les gestes d'attaque et de relâchement aient beaucoup d'influence sur le son piano (voir Bernays et Traube 2014), et une certaine influence au clavecin (voir Paté et al. 2017). Au clavicorde, la liaison entre

11 En fonction des facteurs d'instrument, des modèles et du registre, du goût et de la technique de l'instrumentiste, l'enfoncement au clavier est d'environ 3 à $6 \mathrm{~mm}$ avant le contact de la corde. 
enfoncement, tension et hauteur tonale permet des ornements comme le balancement, 1'appui secondaire ${ }^{12}$ ou des nuances d'attaque, mais révèle également tout défaut de contrôle digital.

Ce jeu continu sur la profondeur du toucher est unique au clavicorde, c'est le seul instrument à clavier qui l'autorise, mis à part les claviers électroniques d'aujourd'hui. Par ce jeu sur l'intonation, le toucher est finalement plus expressif que sur les autres instruments à clavier, au dire des auteurs anciens, instruments pour lesquels attaques et relâchements sont les seuls espaces de jeu.

\section{L'école de Jean-Sébastien Bach}

La seconde moitié du $\mathrm{XVIII}^{\mathrm{e}}$ siècle dans l'aire germanique apparaît comme un véritable âge d'or pour le clavicorde ${ }^{13}$. À partir des années 1750 paraissent en Allemagne plusieurs textes sur le jeu du clavicorde, associés à l'école de J.-S. Bach ${ }^{14}$.

Les rapports de J.-S. Bach et du clavicorde ne reposent que sur peu d'informations directes, même si le clavicorde est de manière évidente un instrument commun à cette époque en Allemagne ${ }^{15}$. La passion de C.P.E. Bach pour le clavicorde est par contre bien connue ${ }^{16}$. C'est par lui, pour une grande part, que la réputation pédagogique du clavicorde se rattache à l'école de son père.

La première source à décrire le jeu de clavier de J.-S. Bach se rencontre dans la méthode de flûte de Jean-Joachim Quantz ([1752]1975) qui l'a personnellement connu et entendu ${ }^{17}$. La mention de Bach $^{18}$ renvoie au texte suivant :

Sur chaque instrument le ton peut être rendu de différentes manières, et en effet de même sur le clavecin, quoiqu'en apparence, on croirait, que tout y dépendît de l'instrument seul, et non pas du joueur. L'expérience prouve que si deux musiciens

12 En Allemand : beben (balancer, vibrer) et Bebung (balancement, vibrato). L'ornementation devient particulièrement raffinée chez C.P.E. Bach et son école, avec la notation du Bebung dans les partitions, et d'autres ornements spécifiques au clavicorde, comme l'appui secondaire d'une note lorsque la touche est déjà abaissée (tragen der Töne).

13 Les traités d'Adlung [1726]1768 et de Walther 1732 donnent une bonne image de la place de l'instrument à l'époque.

14 Ces textes sont cités et analysés, par exemple, dans : Geoffroy-Dechaume 1986, Baillot 1992, Van Delft 2003, Speestra 2004 et Ferran 2015.

15 Le seul document du vivant de Bach qui mentionne le clavicorde est un acte notarié, le don par Jean-Sébastien d'un instrument "3. Clavire nebst Pedal », que l'on peut interpréter comme un clavicorde à deux claviers et pédalier, à son jeune fils Johann Christian. Forkel (1802) affirme que Bach préférait le clavicorde au clavecin, mais ce témoignage est indirect. Pour une discussion récente sur les rapports entre Bach et le clavicorde, on consultera l'ouvrage de Speestra 2004.

16 Rappelons ces phrases célèbres de son Traité (Introduction, première partie, $\S 11$ et $\$ 15$ ) : « C'est au clavicorde qu'un claviériste est le plus exactement évalué », puis " Un bon clavicordiste est un claveciniste accompli, mais non l'inverse ».

17 Antoine Geoffroy-Dechaume a édité le traité de Quantz (avec le flûtiste Pierre Séchet). Il a mis en pratique la méthode de toucher décrite par Quantz et l'a enseignée.

18 Elle se trouve dans l'index de l'ouvrage sous le titre : Jean-Sébastien Bach, sa méthode de mettre les doigts au clavecin XVII-VI-18. 
différents en mérite jouent du même clavecin, les tons seront pour beaucoup meilleurs sous la main du plus habile. On n'en peut donner d'autre raison que celle qui vient de la différente manière de toucher, et il est nécessaire à cet égard, que tous les doigts touchent avec une force égale et avec le juste poids ; que l'on donne aux cordes le juste temps, pour faire leurs vibrations sans obstacle ; qu'on ne les touche pas avec trop de lenteur ; leur donnant au contraire, moyennant une tire, une certaine force qui les fait faire autant de vibrations, qu'il faut pour que le ton dure plus longtemps ; et c'est par-là qu'il faut, autant qu'il est possible, obvier au défaut naturel de cet instrument, qui est que les tons ne peuvent se lier ensemble comme sur d'autres instruments. Il importe aussi de regarder si l'on pousse avec un doigt plus fort qu'avec l'autre. Cela peut arriver, quand on s'est accoutumé, de courber quelques doigts plus en dedans que les autres qu'on tient droit ; ce qui cause non seulement une force inégale dans le jeu, mais empêche aussi d'exprimer les passages rondement, distinctement et agréablement ; et de cette façon là, devant exécuter un roulement de quelques notes qui vont par degré, on ne fait que broncher, pour ainsi dire, sur les notes. Si au contraire on s'accoutume d'abord de courber également tous les doigts, les uns pas plus que les autres, on ne tombe pas si facilement dans cette faute. Outre cela, il faut dans l'exécution de pareilles notes roulantes, ne pas lever brusquement les doigts ; il faut plutôt retirer leur pointe jusqu'au bout antérieur de la touche, et les glisser en bas de cette façon-là ; c'est alors que les passages roulants s'expriment le plus distinctement. J'appuie mon sentiment sur l'exemple d'un des plus habiles joueurs de clavecin, qui avait cette méthode et qui l'enseignait aussi (Quantz 1752, p. 235-236).

Plutôt qu'un mouvement strictement vertical du doigt, Quantz décrit un mouvement rotatif, avec un retrait du doigt vers l'arrière de la touche après le contact. Le doigt est comme tiré en arrière. Quantz parle d'une " tire » pour nommer ce mouvement du doigt. Comme le traité paraît à l'époque à la fois en français et en allemand, la traduction du terme français de "tire " est donnée par Quantz lui-même en allemand par le substantif «Schneller», qui semble apparaître ici pour la première fois. On trouve aussi dans ce texte pour décrire le geste digital les verbes retirer ( ziehen») et glisser («abgleiten»). Quantz mentionne « la force inégale dans le jeu », ce qui semble s'appliquer davantage au clavicorde qu'au clavecin, pour lequel la force exercée sur les touches n'a que peu d'effet sonore.

Un autre témoignage du jeu de Bach est donné par C.P.E. Bach, qui tout comme Quantz a appartenu à la cour de Frédéric II de Prusse. Le nom de J.-S. Bach n'est pas mentionné dans le traité de son fils, mais C.P.E. Bach affirme par ailleurs qu'il n'a eu de maître que son propre père. Une analyse détaillée du traité de C.P.E. Bach ([1753]2009) par Van Delft ${ }^{19}$ (2003) fait apparaître les mots « schnellen » (verbe sous forme source, dont on rencontre diverses formes dérivées dans le texte), "Schnellen »

19 Menno Van Delft pratique la méthode du toucher de Bach. On trouvera facilement sur la toile des images et des enregistrements de son jeu. Van Delft cite dans son article de 2003, sans détailler ni donner les références de toutes les contributions, les noms de F.W. Marpurg (1763), G.S. Löhlein (1765), E.W. Wolf (1785, 1788), D.G. Türk (1789), G.F. Wolff (1799), J.H. Knecht (1800), J.N Forkel (1802), A.E. Müller (1804), F.C. Griepenkerl (1819), J.G. Kaye (1820) et C. Czeny (1830). 
et «Schneller» (substantifs). Le substantif «(der) Schneller» désigne spécifiquement un ornement, qui fait l'objet d'un article particulier de quatre paragraphes dans le chapitre II ("Les ornements »). Le verbe " schnellen " peut être substantivé en " (das) Schnellen ». Les deux versions de ce mot "schnellen » désignent le geste digital, que nous traduisons provisoirement par «tire ». On le trouve dans ce sens à deux reprises dans le traité. La première au sujet des passages rapides (Chapitre I, §90, p. 46) :

... le Schnellen [la tire], une rapide rétraction qui se produit lorsqu'un doigt quitte la touche aussi vite que possible de façon à ce que chaque son soit distinctement perçu. Ce type de passage est aisément réalisé au clavicorde.

La seconde au sujet du trille, pour une double articulation (Chapitre II, §8, p. 73) :

Il est gechnellt [tiré, accéléré] ; après l'attaque, l'articulation supérieure du doigt est rapidement doublée et retirée de la touche aussi vite que possible.

Dans la préface d'un recueil de sonates, Ernst Wilhelm Wolf (1785), ami de C.P.E. Bach donne une description précise de la technique du "Schnellen » (Préface, p. IV) :

On frappe la touche avec un doigt ferme (comme pour jouer une syncope), et on tire immédiatement le doigt en arrière vers le joueur de façon à ce qu'il glisse à l'avant de la touche, et la touche remonte ainsi vivement. Le son, joué sur un bon clavicorde, sonne comme si les consonnes «t'nt» accompagnaient le son; ce «t'nt» fait un meilleur effet que le « $\mathrm{tt}^{\prime}$ » que l'on obtient lorsque l'on quitte la touche sans le glissement ${ }^{20}$.

Cette analogie avec les consonnes indique un contrôle continu de la qualité du son (possible uniquement au clavicorde), et/ou un changement du son transitoire lors du relâchement de la touche (possible également en contrôlant l'étouffoir au clavecin ou au piano). Le mouvement de retrait du doigt permet de varier la tension de la corde et donc la hauteur tonale pendant la note, afin de changer son timbre. Les verbes employés sont glisser (« abgleiten»), et tirer («ziehen»).

La plus longue et la plus connue des descriptions de la technique de clavier de J.S. Bach est apocryphe. Elle se trouve dans l'ouvrage de Johann N. Forkel publié en $1802^{21}$ :

Je n'ai pu m'empêcher de souvent m'étonner que C.F. Emmanuel Bach dans son Essai sur la vraie manière de toucher le clavecin, n'ait pas enfin décrit les caractères qui constituent ce haut degré de netteté dans le toucher de l'instrument ; il possédait en effet lui-même ce toucher qui formait une des grandes originalités de l'exécution de son père. À la vérité, C.P. Emmanuel nous dit bien, dans le chapitre où il traite

20 Ce texte de Wolf est discuté dans Hogwood 1988.

21 Forkel, qui n'a pas connu J.S. Bach, a recueilli souvenirs et documents de la part des élèves de Bach encore vivants, et en particulier de deux de ses fils, W. F. et C. P. E. Bach. Bien que son livre soit finalement publié plus de 50 ans après la mort de Bach, les informations qu'il contient sont généralement considérées comme fiables. 
du style dans l'exécution : "Beaucoup de gens jouent comme s'ils avaient de la glu entre les doigts, et s'attardent sur le clavier, en gardant les touches abaissées au-delà du temps nécessaire. D'autres, essayant de remédier à ce défaut, jouent trop brièvement, comme si les touches leur brûlaient les doigts, ce qui est aussi fautif. Le meilleur est de cheminer entre ces deux extrêmes ». Mais il aurait bien dû nous enseigner les moyens d'atteindre à ce chemin. À son défaut, je vais m'efforcer d'éclaircir cette question, autant du moins que la chose est praticable en dehors de tout enseignement oral.

Bach plaçait la main sur les touches de la façon suivante : les cinq doigts étaient recourbés de manière à faire tomber perpendiculairement leur extrémité sur le clavier, au-dessus duquel ils formaient une ligne parallèle. De cette façon, aucun d'eux n'avait besoin de se rapprocher inégalement de la touche qu'il devait abaisser ; de plus, cette position les mettait à même d'obéir immédiatement au commandement. Voici en détail les conséquences de cette position de la main : 1) les doigts ne peuvent tomber ou bien être jetés sur leurs touches respectives comme il arrive si souvent ; tout au contraire ils sont ainsi posés sur la note avec la pleine conscience de la puissance interne qu'ils ont à développer ; de plus, ils sont dans la possibilité absolue de commander au mouvement ; 2) l'impulsion ou la quantité de pression communiquée à la touche doit être maintenue avec égalité : pour cela, le doigt ne se doit pas lever perpendiculairement de la touche, mais bien plutôt glisser doucement le long de cette touche, en se repliant graduellement vers la paume de la main ; 3) dans cette transition d'une touche à l'autre, la glissade sert à communiquer avec la plus grande rapidité au doigt voisin la quantité exacte de pression employée par le doigt précédent, de telle sorte que les deux sons se trouvent n'être ni détachés ni soudés ensemble. Le toucher n'est alors ni empâté ni trop bref, comme le dit C. P. Emmanuel : "Il est ce qu'il doit être ».

Les avantages de cette position de la main et de ce toucher sont nombreux, soit qu'on les applique au clavicorde ou au clavecin, soit qu'on les applique à l'orgue. Je n'en mentionnerai que les plus importants : 1) la position des doigts ainsi recourbés rend tous leurs mouvements faciles ; ils n'ont aucun effort à faire pour atteindre la touche, sur laquelle ils ont du reste une moindre chance de trébucher, et qu'ils ne peuvent surtout attaquer à coups de poing, toutes choses fort habituelles aux personnes qui jouent avec les doigts tendus ou insuffisamment recourbés ; 2) le fait de replier le bout des doigts, et la rapide transmission de la force d'un doigt à un autre qui en est le résultat, produisent un haut degré de netteté dans l'expression des notes, et les traits rendus de cette manière ont un son plein, égal et brillant. L'auditeur n'a nul besoin de tendre son esprit pour saisir un passage de ce genre. La glissade de l'extrémité du doigt sur la touche avec une pression uniforme laisse à la corde un temps suffisant pour vibrer ; le son est plus beau, et sa prolongation permet à l'artiste de lier convenablement des notes de longue durée sur un instrument si pauvre de son comme est le clavicorde. De plus, ce système a dans son ensemble le très grand avantage d'éviter tout gaspillage inutile de force et toute gêne dans les mouvements. Il paraît que Bach jouait avec un mouvement des doigts si aisé et si peu accentué qu'il était à peine perceptible. Les premières phalanges de ses doigts remuaient seules ; sa main conservait la forme arrondie, même dans les passages les plus difficiles ; les doigts s'élevaient fort peu au-dessus des touches, à peine un peu plus que dans la position du trille ; et dès qu'un doigt cessait d'être employé, il avait soin de le replacer dans sa position réglementaire. Les autres parties de son corps avaient encore bien moins de part à son exécution contrairement à ce qui arrive à 
beaucoup de gens dont la main n'a point une agilité suffisante. Une personne peut néanmoins posséder toutes ces qualités et n'être en définitive qu'un virtuose fort ordinaire sur le clavecin ; de même qu'un homme peut avoir une diction nette et agréable, et n'être qu'un détestable déclamateur. Pour être bon exécutant, il faut encore d'autres qualités tout aussi nécessaires, que Bach possédait au plus haut degré (Forkel 1802, p. 520-522).

On retrouve dans ce texte des réminiscences de ceux de Quantz et C.P.E. Bach. Forkel a pratiqué et enseigné au clavicorde cette technique digitale, comme en témoigne en 1819 un de ses élèves, Friedrich Konrad Griepenkerl, dans sa préface à la Fantaisie chromatique et fugue de $\mathrm{Bach}^{22}$ :

De plus, le clavicorde est de loin préférable au forte-piano pour débuter, toute erreur de toucher est facilement entendue, et tout dépend davantage du joueur que de l'instrument. [...] L'école de Bach demande de la netteté, de l'aisance et de la liberté dans l'interprétation, même des œuvres les plus difficiles, à un degré que l'on ne peut atteindre qu'au moyen de sa méthode particulière de toucher. Ce toucher a été si clairement et précisément décrit par Forkel dans son petit volume « Über J.S. Bachs Leben, Kunst and Kunstwerke » que plusieurs personnes intelligentes, qui ont pris au sérieux ces questions sans se laisser distraire par des préjugés étroits, ont réussi à le maîtriser parfaitement, en l'absence d'exemple ou d'enseignement direct (Griepenkerl 1819, dans Faulkner 1988, p. 63 ; traduction de l'auteur).

Encore plus tardivement, les mêmes conseils pour le piano sont donnés en 1850 par Eduard Eggeling, un élève de Griepenkerl (voir Zapf 2002). Dans la méthode de piano de Herz (1860), le glissement du doigt apparaît comme le moyen pour réaliser un " détaché moelleux ». Mais il n'y a ici plus de référence au clavicorde.

Vers la fin du XVIII siècle, l'intérêt pour le clavicorde, ainsi que celui pour J.-S. Bach, s'étend aussi à la France, en grande partie grâce au prestige de C.P.E. et Johann Christian Bach, et des nombreux musiciens allemands ou alsaciens présents dans la capitale. Jean-Baptiste Grosier note :

À cette occasion je me rappelle une anecdote dont je n'ai jamais cru être dans le cas de faire usage. Un célèbre Compositeur d'Italie vint en Allemagne, prévenu contre le Clavicorde, qu'il regardait comme un instrument imparfait que l'on conservait encore par habitude. Un de ses Compatriotes qui habitait ce pays depuis longtemps, le conduisit chez un grand Maître (Sébast. Bach, père de tous les Bachs, autant que je puis me le rappeler.) Déjà étonné par les préludes de cet habile Professeur sur le Clavicorde, il fut attendri aux larmes par un adagio qui les suivit, attendit à peine la fin du morceau pour se jeter au cou de l'Artiste, \& s'écria : c'est le Roi des Instruments (Grosier 1780, p. 174).

22 Ewald Kooiman a retrouvé et présenté le texte oublié de Griepenkerl (Kooiman 1983, traduction anglaise par Faulkner 1988), témoignage tardif, mais particulièrement éloquent de l'école de Bach. Il a développé une technique digitale apprise aux mêmes sources littéraires, des images de son jeu et des enregistrements se trouvent facilement sur internet. 
Le paradoxe du clavicorde est lui même explicitement mentionné (sous forme de l'antithèse l'avantage... et son inconvénient) par Nicolas-Joseph Hülmandel (1791) :

L'avantage de cette languette [la tangente du clavicorde] est d'augmenter \& d'adoucir le son en appuyant du doigt plus ou moins fort sur la touche, \& son inconvénient est de le hausser ou de le baisser en même temps. [...] L'usage du clavicorde s'est conservé en Allemagne à cause de sa commodité, du peu d'entretien qu'il exige, $\&$ parce que dans ce pays, où l'on a plus qu'ailleurs fait des recherches sur l'art de toucher du clavecin, on a observé que l'exercice du clavicorde est très-propre à perfectionner le tact. La plus légère différence de force dans les doigts y est sensible, \& la moindre irrégularité peut faire un mauvais effet.

L'inconvénient que les languettes de cuivre des clavicordes ont de hausser le son quand on l'augmente, \& de ne pas laisser aux cordes une libre vibration, a fait imaginer de pincer les cordes avec de petits morceaux de plumes qu'on a fixés à des languettes à ressort, enchâssées dans la partie supérieures de petits morceaux de bois minces \& plats nommés sautereaux (Hülmandel 1791, p. 285-286).

Hülmandel n'est pas éloigné du cercle de Bach, qu'il a pu fréquenter soit par un contact direct avec C.P.E. Bach, soit par des contacts indirects à travers son éducation musicale à Strasbourg ${ }^{23}$.

\section{Toucher poussé et toucher tiré}

Le même geste est décrit par les textes anciens à l'aide de plusieurs mots, en français et en allemand. Quantz, qui n'était pas de langue maternelle française, utilise le substantif «tire », et la périphrase « retirer leur pointe jusqu'au bout antérieur de la touche ». Le mot «tire» est d'un emploi rare à l'époque, comme il l'est aujourd'hui ${ }^{24}$. « Retirer la pointe » correspond pour Quantz à «zurücke ziehen ${ }^{25}$ », soit en français « tirer en arrière », un mot repris par Wolf (1785). Mais en allemand, sa langue maternelle, Quantz introduit un mot nouveau " (der) Schneller" à l'endroit où il emploie " tire " en français. Ce mot est repris par C.P.E. Bach, dans un sens différent, celui d'un ornement. C.P.E. Bach utilise " (das) Schellen » pour décrire le geste de la " tire ». Les mots "schnellen/Schneller/Schnellen » évoquent un geste rapide, une accélération, le claquement du doigt, le claquement du fouet et les sons associés à ces actions. Quantz et Wolf emploient aussi le verbe glisser («abgleiten»). C.P.E. Bach emploie le verbe effleurer ou caresser («schmeicheln $»)^{26}$.

23 Hülmandel imagine (à tort) que le sautereau du clavecin a été inventé pour résoudre le paradoxe du clavicorde : son échappement laisse à la corde une libre vibration (plus de contact avec le doigt à travers la tangente et la touche).

24 Le Dictionnaire de l'Académie française (édition de 1762, p. 836) contient néanmoins la vedette « tire». C'est un nom féminin : «Il n'est d'usage que dans ces phrases, voler à tire d'aile, pour dire, voler aussi rapidement qu'il est possible. Et, tout d'une tire, pour dire, sans discontinuation, tout de suite. Ce dernier n'est que du style familier. »

25 «[...] sondern die Spitzen derselben vielmehr, auf dem vordersten Theile des Tasts hin, nach sich zurücke ziehen, bis sie vom Taste abgleiten» (Quantz [1752]1975, XVII-VI-18).

26 C.P.E. Bach mentionne (Introduction, §15, p. 11) : Le clavicordiste prend trop l'habitude d'effleurer [schmeicheln] les touches. 
Nous proposons de réemployer le premier terme de Quantz et d'introduire la locution toucher tiré. Le toucher tiré correspond au mouvement de l'école de Bach. Le doigt glisse dans le plan horizontal tout en abaissant la touche, avec un retrait de la pointe du doigt vers la paume de la main, jusqu'au relâchement de la touche. C'est un mouvement rond du doigt, qui se développe dans un plan sur une trajectoire d'allure circulaire. L'attaque de ce mouvement est représentée sur la figure 1 et dans l'extrait vidéo 1 .

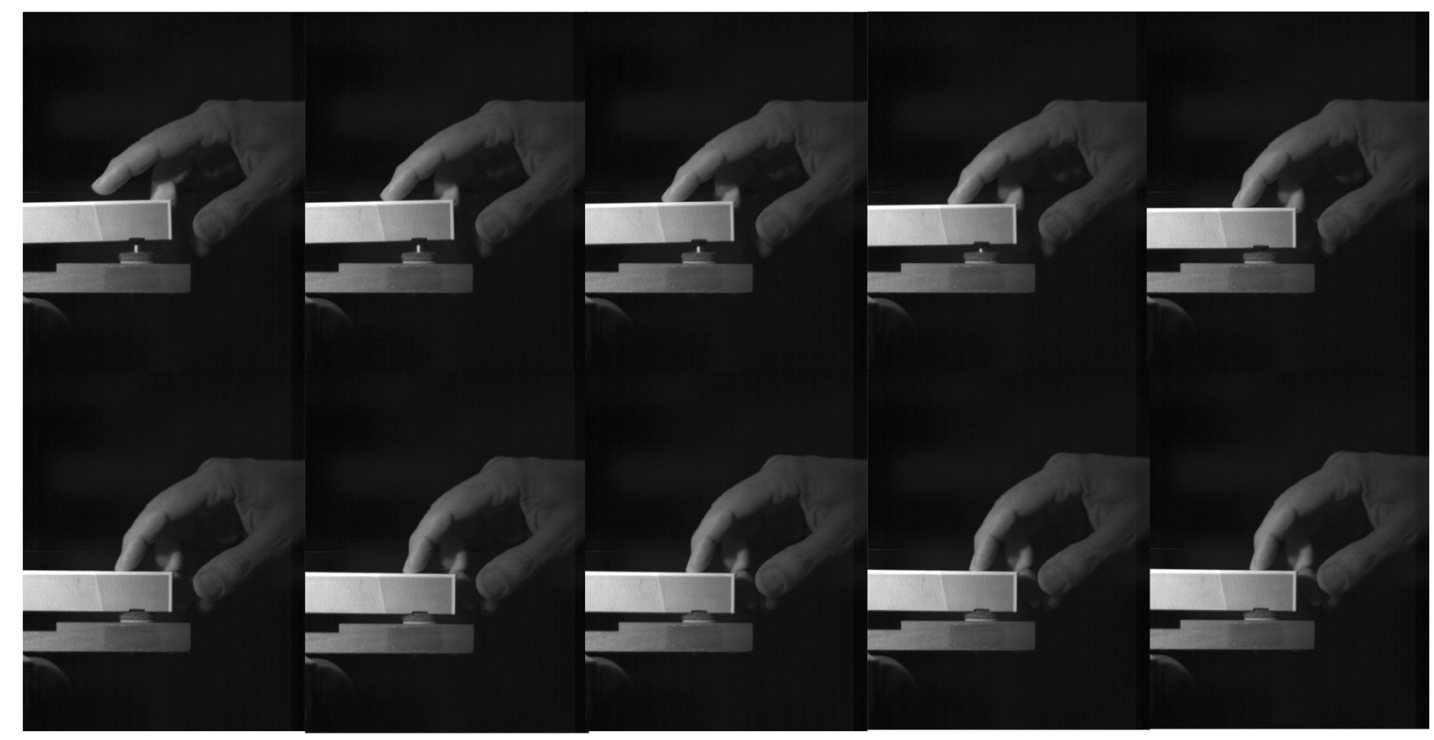

Figure 1 : Attaque de la note avec le toucher tiré, filmé en caméra rapide. Une touche modèle de piano est utilisée pour la visualisation.

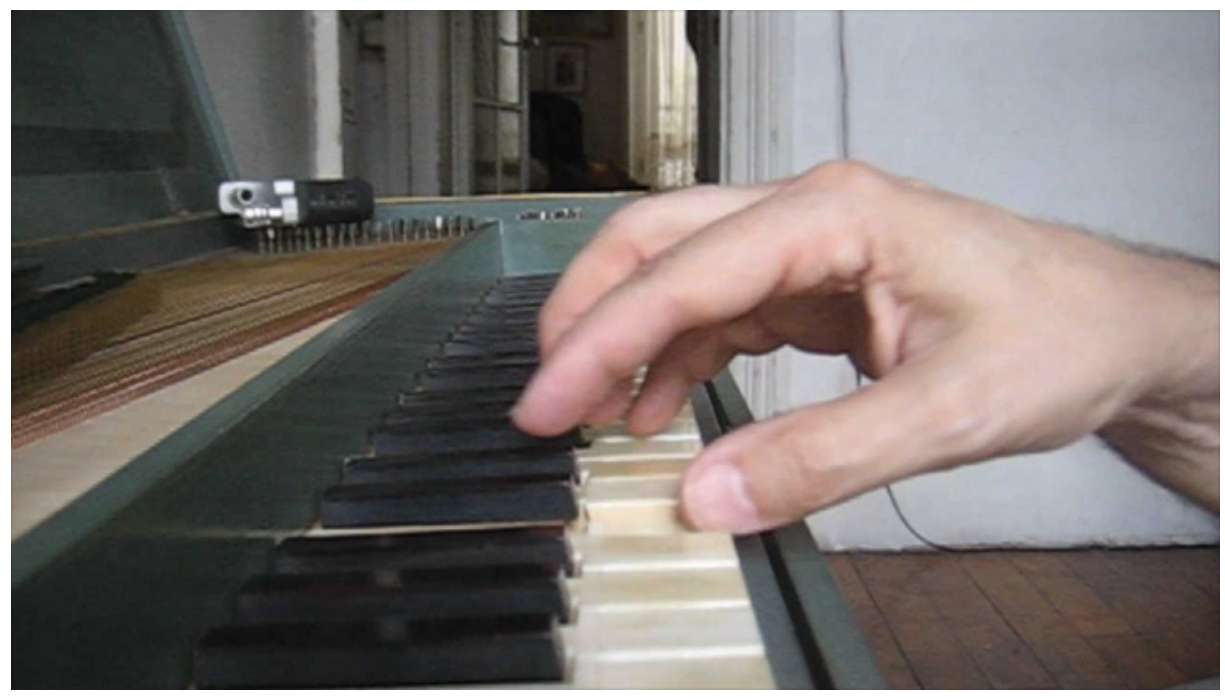

Extrait vidéo 1: Attaque de la note avec le toucher tiré, pour les cinq doigts, main droite (tiré, poussé, tiré, poussé) puis main gauche (tiré, poussé, tiré, poussé).

$\mathrm{Au}$ contraire, le toucher poussé correspond à un mouvement vertical du doigt, un abaissement vers le bas de la touche C'est un mouvement droit du doigt, qui se développe sur une droite, dans une trajectoire rectiligne. L'attaque de ce mouvement est représentée sur la figure 2 et dans l'extrait vidéo 1. 


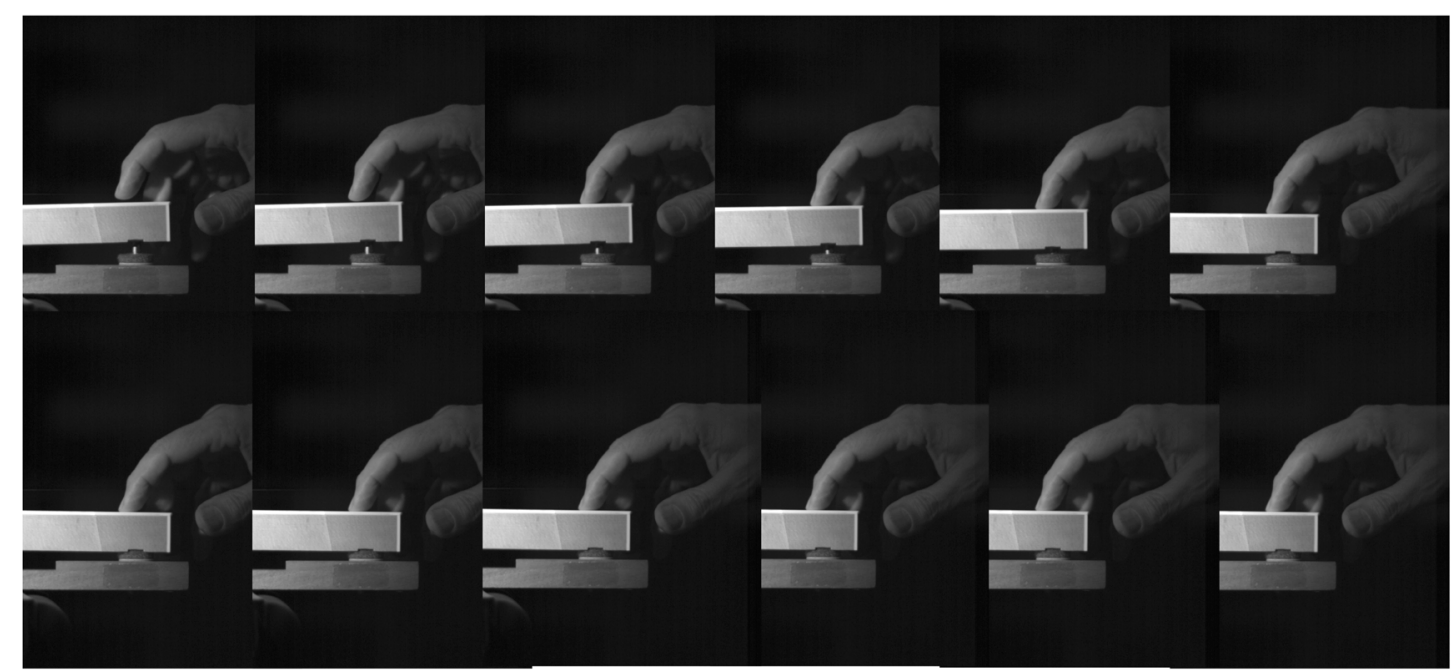

Figure 2: Attaque de la note avec le toucher poussé, filmé en caméra rapide. Une touche modèle de piano est utilisée pour la visualisation.

\section{LA MÉCANIQUE DU CLAVICORDE ET LES RELATIONS ENTRE LA JUSTESSE D'INTONATION}

\section{ET LA FORCE DU JEU}

La mécanique du clavicorde est très simple. Le clavier est composé d'un balancier qui pivote autour de la pointe de balancier. À l'extrémité externe de l'instrument se trouve la touche, sur laquelle le doigt exerce la pression requise ayant pour effet d'abaisser cette partie. À l'extrémité interne se trouve la tangente, qui frappe le chœur (une paire) de cordes par relèvement correspondant de cette autre partie. Le mouvement exercé sur la touche par le doigt est transmis mécaniquement par l'intermédiaire du balancier et de la tangente jusqu'à la corde aussi longtemps que dure la pression du doigt sur la touche. Au relâchement de la touche, un étouffoir absorbe la vibration des cordes et le son.

Les cordes du clavicorde vont en général par paire, ce qui est préférable à la fois pour le son et le toucher. Le son d'une double corde est plus complexe et dure plus longtemps en raison du couplage des deux cordes au niveau du chevalet ${ }^{27}$. La résistance de deux cordes est plus grande que celle d'une seule, ce qui permet au musicien de sentir une plus grande fermeté ou contre-force au niveau du doigt lorsqu'il maintient la touche enfoncée, assurant ainsi un meilleur contrôle.

Les principaux paramètres sonores étudiés ici sont la fréquence fondamentale et le niveau de pression acoustique, en relation avec la vitesse de la tangente. Pour le musicien, cela correspond principalement à la hauteur perçue et à la nuance dynamique. La dynamique du clavicorde induit principalement une variation de SPL, et marginalement une variation spectrale ${ }^{28}$.

\footnotetext{
27 Ce qui est bien connu pour le piano. Voir Weinreich 1977.

28 Contrairement au piano, pour lequel la richesse spectrale varie avec la dynamique. Voir Alessandro 2010b.
} 
Hauteur de tangente et $\mathrm{F} 0$

Toutes les notations correspondent à la figure 3, une vue en coupe qui schématise le fonctionnement du clavicorde.

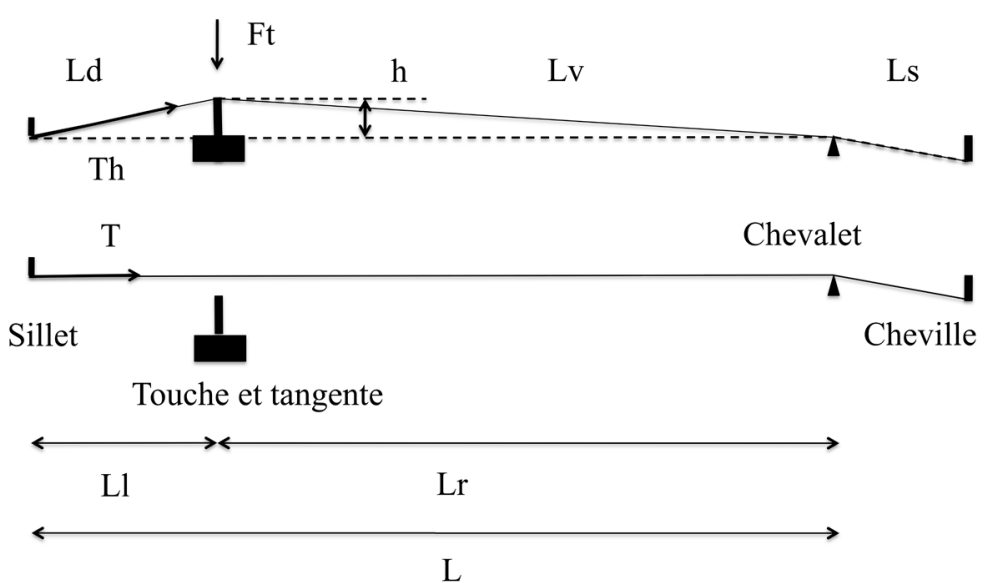

Figure 3 : Géométrie des cordes, avec et sans élévation de la tangente.

La corde vibre entre le chevalet et la tangente. La partie entre pointe d'accroche et tangente ne sonne pas. La partie entre chevalet et cheville vibre par sympathie. Avec les conventions suivantes, pour la figure 3 :

$L \quad$ longueur de la corde au repos

$L_{v} \quad$ longueur vibrante, tangente à la hauteur $h$

$L_{d} \quad$ longueur étouffée, tangente à la hauteur $h$

$L_{r} \quad$ longueur vibrante, au repos

$L_{l} \quad$ longueur étouffée, au repos

$L_{s} \quad$ longueur sympathique

$h \quad$ hauteur de tangente

$T \quad$ tension au repos

$T_{h} \quad$ tension, tangente à hauteur $h$

$F_{t} \quad$ force totale sur la tangente à hauteur $h$

$F_{v} \quad$ force sur la tangente à hauteur $h$, de la partie vibrante

$F_{d} \quad$ force sur la tangente à hauteur $h$, de la partie étouffée

Pour une corde idéale, F0 est donnée par la formule de Mersenne :

$$
F 0=\frac{C_{s}}{2 L_{v}}=\frac{1}{d L_{v}} \sqrt{\frac{T_{h}}{\pi \rho}}
$$

avec $C_{s}$ la vitesse de propagation dans la corde $(\mathrm{m} / \mathrm{s}), \rho(\mathrm{kg} / \mathrm{m} 3)$ la densité, $d(\mathrm{~m})$ le diamètre de la corde, $T_{h}$ sa tension. Les cordes du clavicorde sont fines et relativement peu tendues, elles montrent beaucoup moins d'inharmonicité que celles du piano ${ }^{29}$. Cette simple formule semble donc une approximation acceptable. 
Lorsque l'on abaisse la touche, la corde monte au-dessus de sa position de repos, ce qui entraîne un accroissement de tension, donc de $F 0$. Soit $\lambda$ le module d'élasticité de la corde. Par rapport à la position de repos, la corde soulevée par la tangente d'une hauteur $h$ s'accroît d'une longueur $L_{v}+L_{d}-L$, ce qui donne un accroissement de tension $\Delta T_{h}$ de la corde :

$$
\Delta T_{h}=\frac{\lambda\left(\sqrt{L_{r}^{2}+h^{2}}+\sqrt{L_{l}^{2}+h^{2}}-L\right)}{L}
$$

par un développement limité $\left(h \ll L_{r}\right.$ et $\left.h \ll L_{l}\right)$ on obtient :

$$
\Delta T_{h} \approx \frac{\lambda\left(L_{r}\left(1+h^{2} / 2 L_{r}^{2}\right)+L_{l}\left(1+h^{2} / 2 L_{r}^{2}\right)-L\right)}{L}=h^{2} \frac{\lambda}{2 L_{r} L_{l}}
$$

Ce qui donne :

$$
\begin{gathered}
F 0=\frac{1}{d L_{v}} \sqrt{\frac{T+\Delta T_{h}}{\pi \rho}} \approx \frac{1}{d L_{r}} \sqrt{\frac{T}{\pi \rho} \sqrt{1+h^{2} \frac{\lambda}{2 L_{r} L_{l} T}}} \\
F 0 \approx \frac{1}{d L_{r}} \sqrt{\frac{T}{\pi \rho}}\left(1+h^{2} \frac{\lambda}{4 L_{r} L_{l} T}\right)(1)
\end{gathered}
$$

pour une corde de $L_{l}=0,15 \mathrm{~m}, L_{r}=0,84 \mathrm{~m}$, en laiton $\left(\rho=8470 \mathrm{~kg} / \mathrm{m}^{3}\right)$ de diamètre $0,51 \mathrm{~mm}$, qui sonne do $=123 \mathrm{~Hz}$, la tension est de 73,96 N. Avec un module de Young de $\left(103,10^{9} \mathrm{~Pa}\right)$, une montée de $1 \mathrm{~mm}$ porte $F 0$ à $123,07 \mathrm{~Hz}$, soit 1 Cent, une augmentation non perçue ${ }^{30}$. Une hauteur de $5 \mathrm{~mm}$ correspond à $124,72 \mathrm{~Hz}$, soit 24 Cent, proche du $8^{\mathrm{e}}$ de ton, ce qui est nettement perceptible.

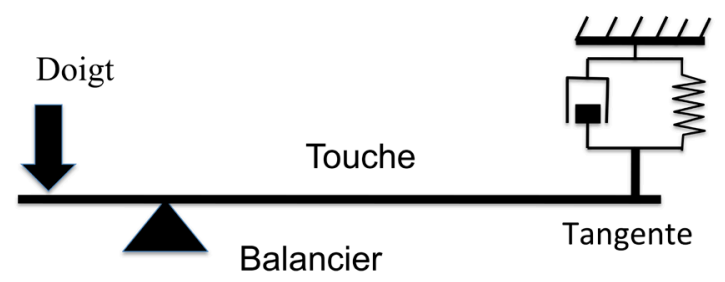

Figure 4 : Effet de ressort de la corde sur la tangente et le doigt.

Lorsque la tangente touche le chœur de cordes, une résistance est ressentie ${ }^{31}$. La figure 4 schématise cet effet de ressort amorti de la corde qui s'oppose au mouvement du doigt. Pour une tangente de hauteur $h$, la force exercée par les cordes est (en utilisant la figure 3) :

$$
F t=h T_{h}\left(\frac{L_{v}+L_{d}}{L_{v} L_{d}}\right)=h T_{h} \frac{\sqrt{L_{r}^{2}+h^{2}}+\sqrt{L_{l}^{2}+h^{2}}}{\sqrt{L_{r}^{2}+h^{2}} \cdot \sqrt{L_{l}^{2}+h^{2}}}
$$

En reprenant l'exemple précédent, pour une paire de cordes, la force sur la tangente est d'environ 0,65 $\mathrm{N}$ par $\mathrm{mm}$ de hauteur, dans la partie élastique de la réponse de la 
corde. Cette partie élastique n'excède pas 7 ou $8 \mathrm{~mm}$ : ensuite la résistance devient très importante, appuyer davantage demanderait beaucoup de force et risquerait d'abimer l'instrument (déformation plastique). On peut négliger dans ce calcul la tension supplémentaire due à l'élévation de la tangente $\Delta T_{h}$ (le calcul donne une variation de $5 \%$ environ).

Vitesse de la tangente et SPL

Dans une étude précédente, nous avons montré que l'intensité du son (sPL en dB) dépend linéairement du logarithme de la vitesse de la tangente au moment de l'impact avec la corde. L'intensité sonore dépend essentiellement de la force exercée par la corde sur le chevalet. $Z 0=T / C_{s}$ étant l'impédance caractéristique de la corde, et $y$ la vitesse de la tangente à l'impact, la force exercée par la corde sur le chevalet (et donc sur la table d'harmonie) est donnée par (voir Alessandro 2010a, équation 8) :

$$
F_{s}=2 Z 0 \dot{y}(t)
$$

La force au chevalet, et donc l'intensité sonore, dépendent ainsi à la fois de la tension de la corde et du rapport entre vitesse de la tangente à l'impact et vitesse de propagation dans la corde (et non de la longueur de la corde ou d'autres facteurs). Après l'impact initial, la vitesse de la tangente décroît de façon exponentielle (voir Thwaites et Fletcher 1981; Alessandro 2010a).

\section{Hauteur de tangente, vitesse et intonation}

F0 dépend de la hauteur de la tangente. Cette hauteur dépend de la vitesse maximale d'impact $V p$. Si la décroissance de la vitesse est exponentielle après l'impact (avec une constante de temps $a$ ) :

$$
y(t)=V p e^{-a t}
$$

la hauteur de la tangente au temps $t$ est :

$$
h(t)=\frac{V_{p}}{a}\left(1-e^{-a t}\right)
$$

L'élasticité de la corde agit comme un ressort s'opposant à l'enfoncement du doigt, qui impose cette forme à la décroissance de la vitesse. Comme $e^{-a t}$ tend vers $0, h$ est proportionnel à $V p$ après l'excitation de la corde, en l'absence de mouvement supplémentaire du doigt. Cette situation correspond à ce que nous dénommons ici « toucher poussé " : le doigt abaisse la corde avec une certaine vitesse (en visant une certaine dynamique ou intensité sonore) par un mouvement vertical, et ensuite maintient simplement la touche abaissée. C'est le mouvement le plus ordinaire au clavier.

En reportant $h(t)$ dans l'équation (1), il apparaît que l'accroissement de $F 0$ en fonction de la dynamique du jeu dépend essentiellement du carré de la vitesse d'impact. Il y a donc un lien intrinsèque entre justesse et force : produire un son fort, augmenter le sPL, c'est abaisser vite la touche, et cela implique, sous l'hypothèse d'un mouvement simple d'abaissement du doigt, le toucher poussé, d'augmenter le déplacement de 
la corde $h$, donc sa hauteur musicale F0. Une évolution plus complexe de la vitesse après l'impact pourrait permettre un contrôle plus différencié, car après tout, le doigt contrôle en permanence la hauteur de la corde. La lecture des sources historiques sur le jeu du clavier nous a donné des descriptions de gestes plus complexes, comme le toucher tiré au clavicorde.

Mesures AVEC TOUCHER POUSSÉ ET TOUCHER TIRÉ

\section{Instrument étudié}

L'instrument étudié a été construit en 1980 par Frédéric Bal (atelier d'Anthony Sidey) à Paris. Ce clavicorde libre (toutes les notes ont une paire de cordes) est inspiré32 d'un modèle allemand de la seconde moitié du XviII siècle conservé au musée de la musique de Paris (E.980.2.666), qui pourrait être rattaché à l'école de Johann Henrich Silbermann (Strasbourg). L'instrument, plus petit que le modèle et qui ne peut être considéré comme un facsimilé, est d'une qualité musicale de tout premier ordre. La figure 5 une image de l'instrument ainsi que ses principales dimensions.

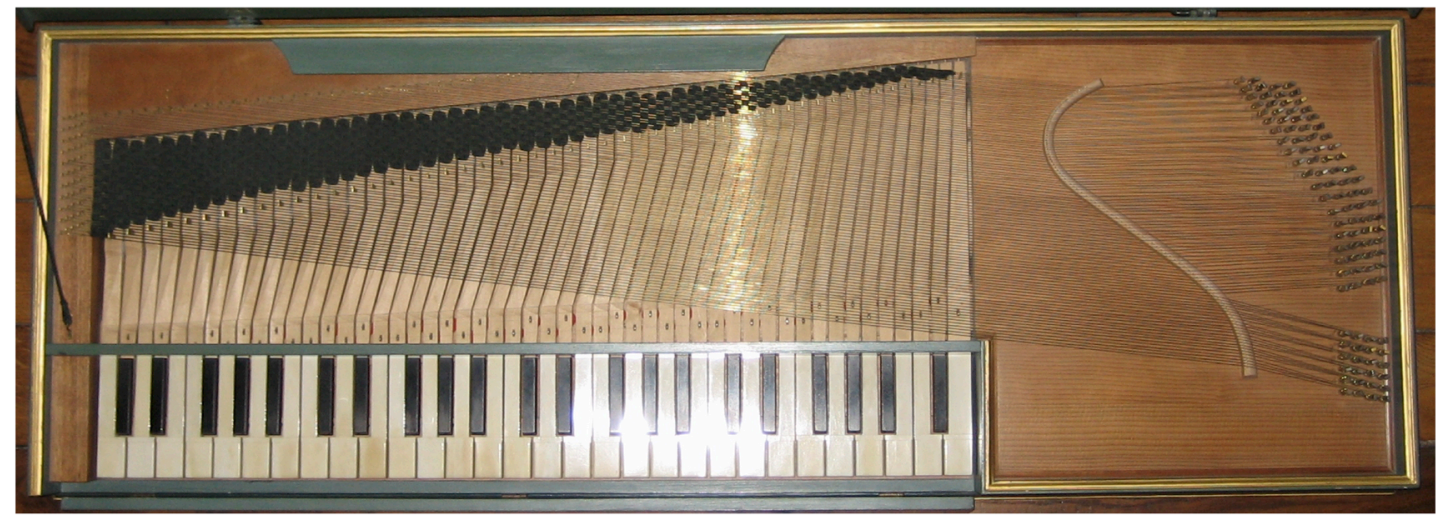

\begin{tabular}{|l|l|}
\hline Étendue & C-d3, do1-ré5 51 notes \\
\hline Cordage & Doubles cordes, laiton \\
\hline Table d'harmonie (mm) & $316 \times 346$ \\
\hline Dimensions (mm) & $1068 \times 361 \times 111$ \\
\hline $\begin{array}{l}\text { Diapason (la) et } \\
\text { tempérament }\end{array}$ & $415 \mathrm{~Hz}$, égal \\
\hline C-do1 (mm) & 895 \\
\hline c-do2 (mm) & 692 \\
\hline c1-do3 (mm) & 441 \\
\hline c2-do4 (mm) & 229 \\
\hline c3-do5 (mm) & 111 \\
\hline
\end{tabular}

Figure 5 : L'instrument étudié, clavicorde libre de Frédéric Bal (1980).

32 Les proportions et les détails de facture sont respectés, mais l'instrument est beaucoup plus petit : $1,068 \mathrm{~m}$ environ de long contre 1,367 $\mathrm{m}$ dans l'original. 


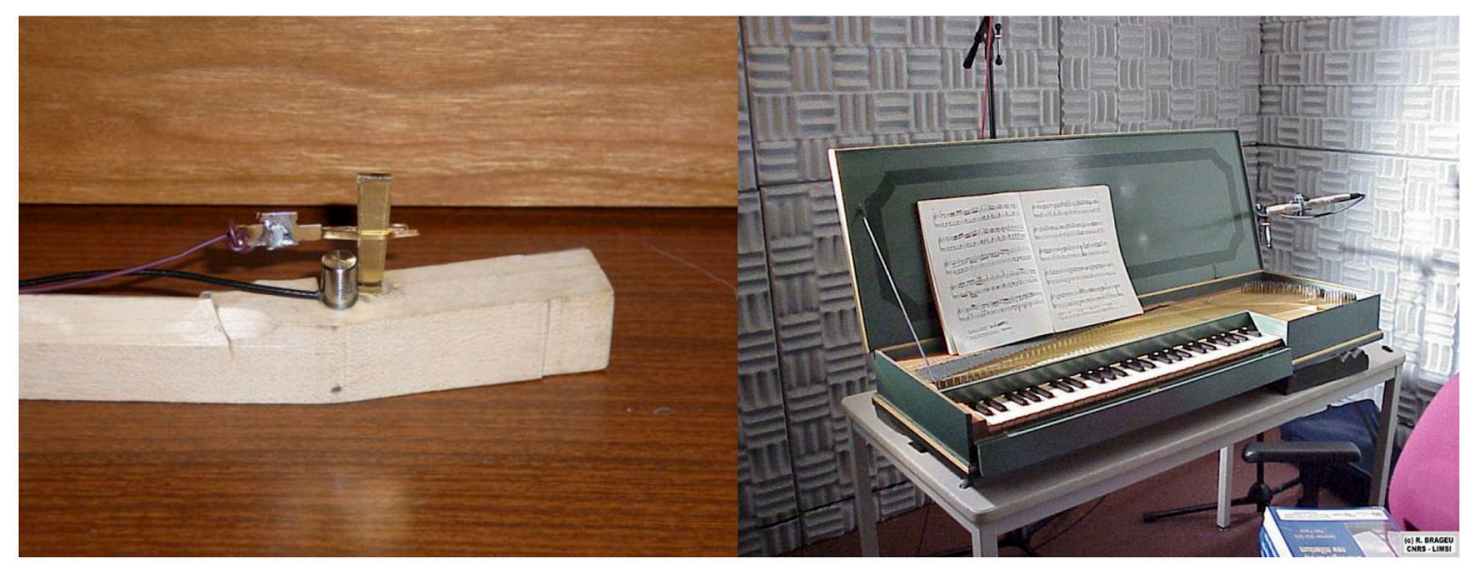

Figure 6 : À gauche, accéléromètre et contact près de la tangente. À droite, placement du microphone à $30 \mathrm{~cm}$ au centre de la table d'harmonie, en cabine d'enregistrement.

\section{Méthodologie de mesure}

L'instrument est placé dans une cabine insonorisée et quasi-anéchoïque. La figure 6 montre le dispositif de mesure installé sur l'instrument. Quatre signaux synchrones sont enregistrés numériquement ${ }^{33}(48 \mathrm{kHz} / 16$ bits) :

1. Le signal acoustique (microphone de mesure omnidirectionnel DPA 4006, carte-son RME Fireface 400), avec le microphone placé à une distance de $30 \mathrm{~cm}$ du centre de la table d'harmonie);

2. Le signal de contact corde/tangente pour la première corde. Ce signal est binaire : 0 s'il n'y a pas de contact ou 1 s'il y a contact. Les cordes et les tangentes étant en métal, un courant électrique est envoyé à la cheville et récupéré à la tangente, lorsqu'il n'y a pas de contact, il n'y a pas de signal, car le circuit est ouvert. Ce signal est porté sur la figure 7 avec la vitesse de la tangente (barre bleue). Le début du contact correspond au maximum de la vitesse. Lorsque l'on relâche la touche, la tangente et la corde se séparent, l'arrière du balancier retombe, et rebondit légèrement, comme on le voit sur le signal de vitesse ;

3. Le signal de contact corde/tangente pour la seconde corde ;

4. La vitesse de la tangente est mesurée avec un accéléromètre miniature B\&K 4374, et un amplificateur de charge B\&K Nexus. La masse de l'accéléromètre est faible (moins de $0,75 \mathrm{~g}$ ) comparée à celle de la touche (environ $20 \mathrm{~g}$ ), ce qui permet de négliger son effet sur les mesures.

33 Cette méthodologie de mesure est appliquée à quatre clavicordes dans Alessandro, Katz et Boudet 2006, Alessandro, Besnainou et Ginieis 2008. Les covariations de F0, sPL et Vp sont présentés dans Alessandro 2016. 

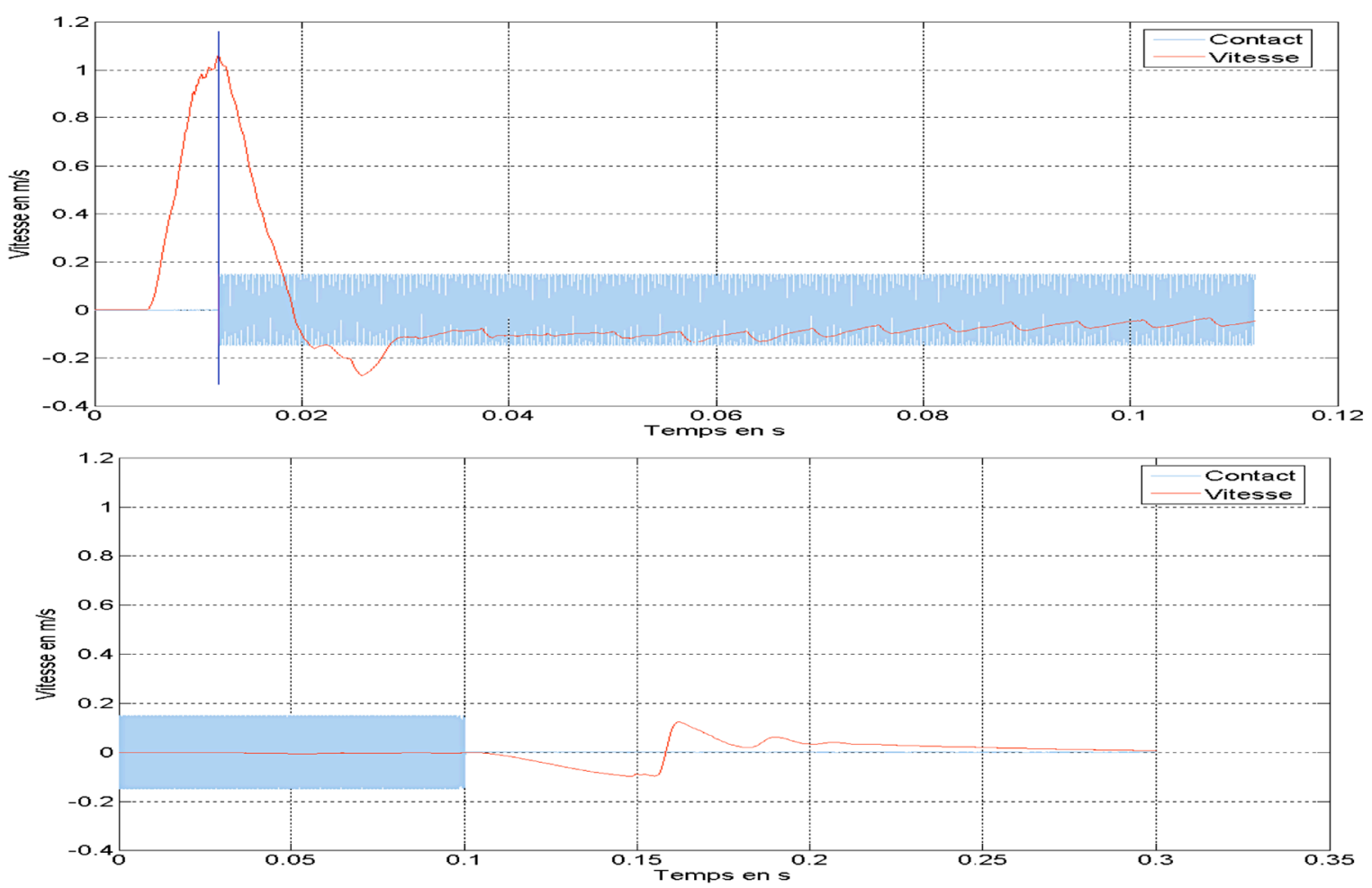

Figure 7 : Signal de vitesse de la tangente et signal de contact corde/tangente (barre bleue), à l'attaque et au relâchement de la note.

Plusieurs quantités sont calculées à partir de ces signaux :

1. Pour le signal de vitesse, le pic de vitesse $V p$ au moment de l'impact semble la mesure la plus représentative du mouvement. Cette mesure représente, à un facteur de levier près ${ }^{34}$, la vitesse verticale maximale du doigt. Elle est obtenue comme le maximum de la vitesse, c'est-à-dire la vitesse au moment de l'impact corde/tangente, au début du signal de contact (voir la figure 7);

2. L'intensité sonore, SPL, est calculée sans pondération. Il s'agit de la valeur en décibels de la moyenne quadratique du signal de pression, avec une pression de référence de $20 \mu P a$. Le temps d'intégration pour la moyenne est de $125 \mathrm{~ms}$. Ce temps assez court est choisi afin de donner davantage d'importance au début du son, en raison de son extinction exponentielle ;

3. F0 est calculé à partir du signal acoustique ${ }^{35}$ toutes les $0,7 \mathrm{~ms}$. Ces valeurs sont moyennées sur une fenêtre de $250 \mathrm{~ms}$, afin de mesurer la hauteur perçue au début de la note.

34 Le levier est dû à la différence de longueurs entre tangente et pointe de balancier d'une part, et doigt et pointe de balancier d'autre part.

35 En utilisant l'algorithme YIN. Voir Cheveigné et Kawahara 2002. 
Toucher poussé
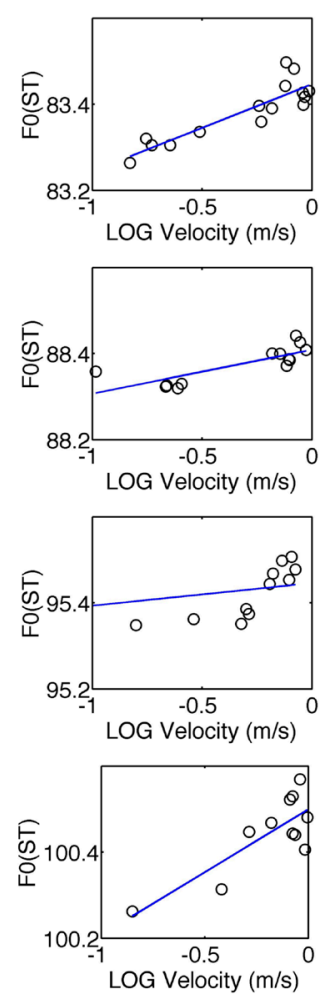
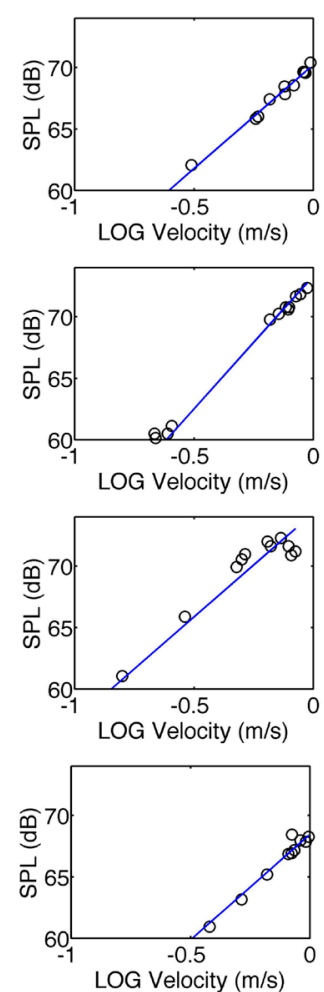

Toucher tiré
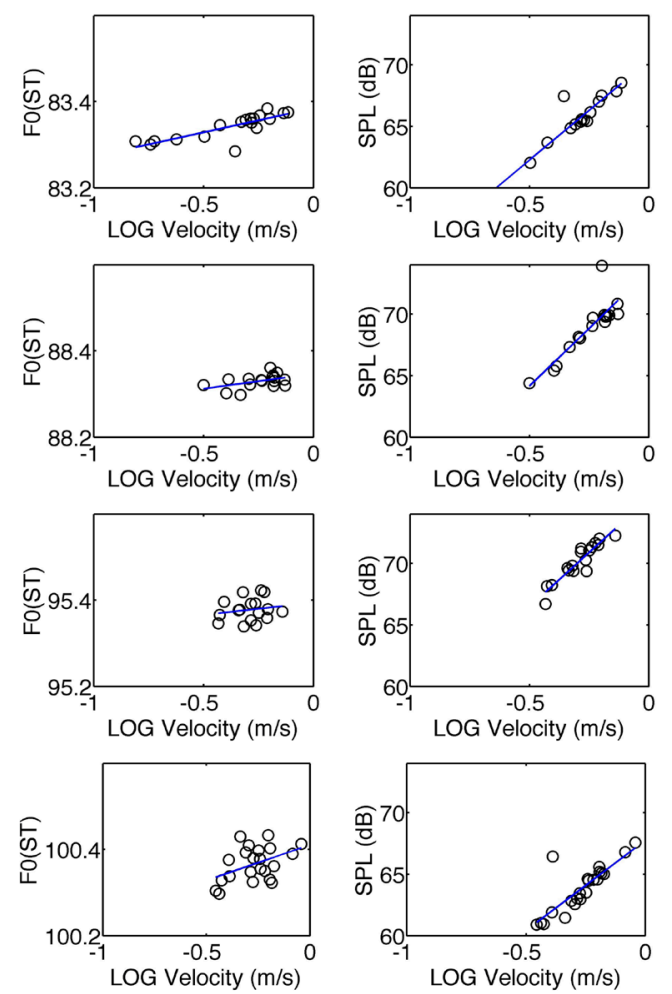

Figure 8: Toucher poussé (à gauche) et tiré (à droite), F0 (demi tons) et SPL (dB) en fonction du logarithme de V pour les notes $\mathrm{do}^{2}, \mathrm{fa}^{2}, \mathrm{fa}^{3}, \mathrm{do}^{3}$.

Toucher poussé

Le toucher poussé correspond au toucher ordinaire, le doigt abaissant la touche puis la maintenant abaissée, avant de la relâcher. Le protocole de mesure, pour chaque touche, consiste à jouer une douzaine de notes dans toute la gamme dynamique de l'instrument, du plus doux au plus fort. Chaque note est maintenue jusqu'à l'extinction complète du son. Deux expérimentateurs ayant une pratique du clavier ont réalisé les mesures.

La figure 8, à gauche montre $F 0$ (demi-tons, intégration $250 \mathrm{~ms}$ ) et SPL (dB, intégration $125 \mathrm{~ms}$ ) en fonction du logarithme de $V p$ (maximum) pour les notes $d o^{2}, f a^{2}, f a^{3}, d o^{3}$. Les mesures ont été effectuées pour toutes les notes de l'instrument, mais un petit sous-ensemble seulement est représenté ici, pour des raisons de place. Ces résultats montrent un bon accord avec la théorie. Pour ce qui est de la dynamique, une dépendance de type linéaire apparaît entre le logarithme de $V p$ et le sPL en $\mathrm{dB}$. La pente de cette relation varie d'une note à l'autre. Cette pente pourrait donner une indication de la gamme dynamique possible sur un instrument particulier et une note particulière, et finalement de la qualité dynamique d'un instrument.

Le logarithme de $F 0$ (demi-tons) est représenté en fonction du logarithme de $V p$ dans la figure 8 pour les mêmes notes. La hauteur des tangentes n'a pas été mesurée directement dans ce travail (un accéléromètre n'étant pas bien adapté pour cela). 
Cependant l'équation (1) montre une dépendance de $F 0$ en racine carrée du pic de vitesse. Donc la hauteur musicale (sur échelle logarithmique) doit dépendre à un facteur 2 près du logarithme du pic de vitesse.

Cette relation linéaire est bien observée dans les mesures, avec cependant moins d'évidence que la relation entre SPL et $V p$. Les résultats précédents montrent que la vitesse des tangentes agit en même temps sur l'intensité sonore et sur l'intonation. Cette dépendance, avec des coefficients variés, implique une contrainte dans le jeu de l'instrument : abaisser rapidement la touche implique de produire un son non seulement de forte intensité, mais également de hauteur tonale élevée, au moins à l'attaque ${ }^{36}$.

\section{Toucher tiré}

Le toucher tiré de l'école de Bach, décrit dans les paragraphes précédents, a fait l'objet d'une seconde série de mesures afin d'étudier l'influence du musicien sur le son du clavicorde, telle qu'elle est décrite dans la littérature ancienne. La consigne était, comme précédemment, de jouer une note avec autant de niveaux dynamiques différents que possible, en contrôlant au mieux la justesse de la hauteur tonale désirée ${ }^{37}$.

Des mesures de vitesse, intensité et intonation en utilisant les deux types de touchers sont portées dans la figure 8 à droite pour les quatre mêmes notes sur le clavier $\left(d o^{2}, f a^{2}, f a^{3}, d o^{3}\right)$. Les résultats montrent que des dynamiques comparables sont obtenues avec les deux types de toucher pour une même note : les deux types de toucher permettent de jouer avec des vitesses comparables, et obtiennent des intensités sonores comparables. En effet, l'intensité sonore dépend peu de l'évolution du geste après l'impact, mais essentiellement de la vitesse au moment de l'impact. C'est elle qui détermine (en rapport avec la vitesse de propagation dans la corde), l'angle de la déformation initiale de la corde, et donc la force du son.

La variation sonore est continue tant que le son est audible. Donc une évolution de la pression sur la touche peut changer la hauteur perçue, ce que l'on constate bien sur les mesures. Alors que les intensités sonores sont comparables, du point de vue de l'intonation, les valeurs de $\mathrm{FO}$ sont plus basses pour le toucher tiré que pour le toucher poussé. Rappelons que les valeurs de FO sont obtenues comme la moyenne sur le début de la note de la fréquence fondamentale, donc une évolution de la tension peut influer sur la valeur de F0. Le mouvement circulaire associé au retrait du doigt lors de la « tire » amène un léger relâchement de la tension de la corde. Il semble donc que ce toucher particulier permette effectivement de limiter la hausse de l'intonation tout en gardant l'intensité sonore : c'est une réponse au paradoxe du clavicorde.

36 Les mêmes mesures ont été réalisées pour quatre instruments, et montrent des tendances identiques, même si les résultats varient d'un instrument à l'autre et d'une note à l'autre pour le même instrument (Alessandro 2016).

37 Les enregistrements ont été réalisés par l'auteur, qui a spécifiquement étudié cette technique digitale avec Antoine Geoffroy-Dechaume entre 1984 et 1986. 


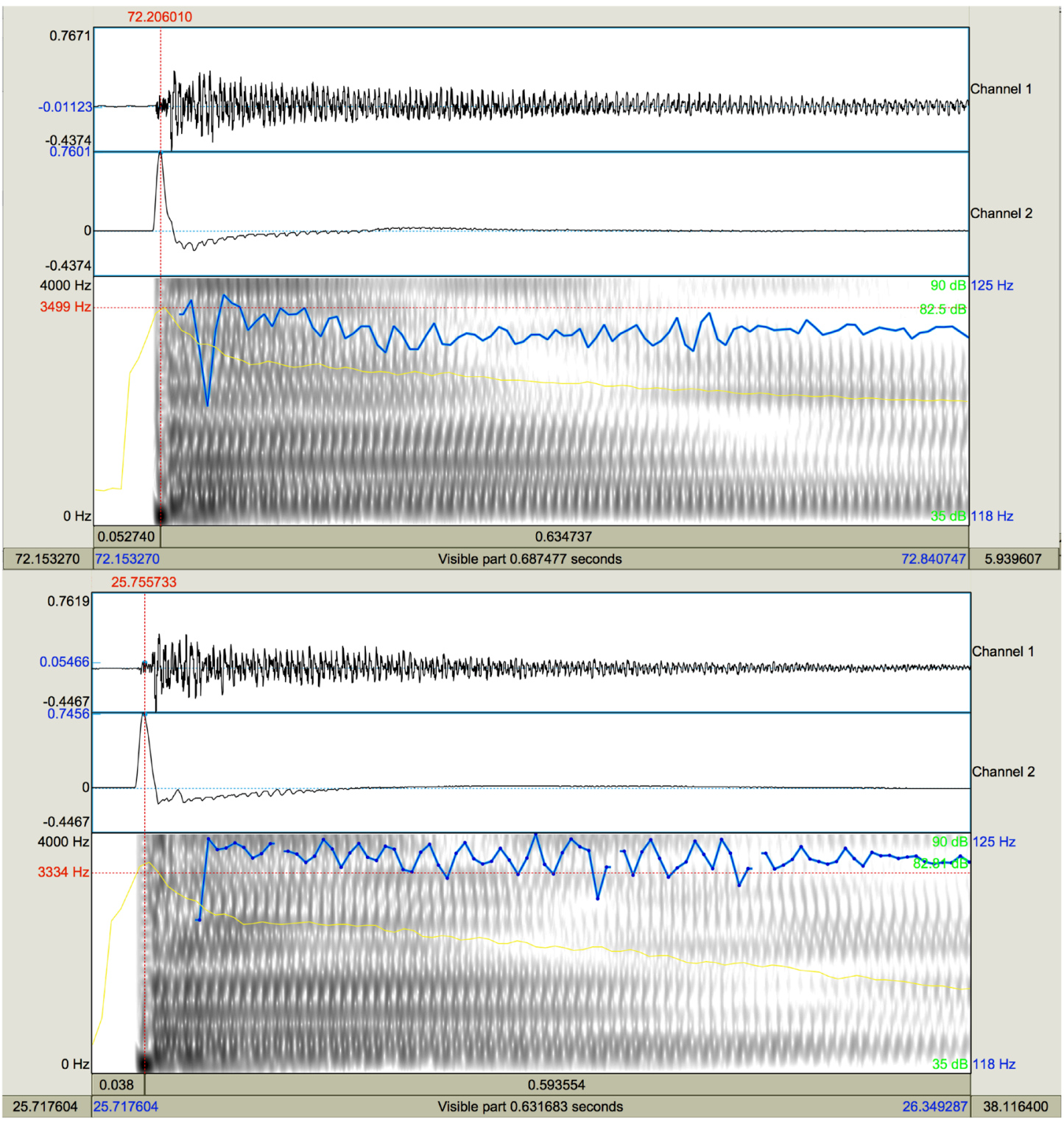

Figure 9: Do², toucher tiré (en haut) et toucher poussé (en bas). Signal acoustique, vitesse de la tangente, spectrogramme avec la courbe d'intensité instantanée (en jaune) et la fréquence fondamentale (en bleu).

Les figures 9 et 10 montrent dans chaque panneaux le signal acoustique (en haut), la vitesse de la tangente (au milieu), le spectrogramme (en bas), l'intensité sonore (en jaune sur le spectrogramme) et $F 0$ (en bleu, sur le spectrogramme) pour les notes $d o^{2}$ et $f a^{3}$ avec le toucher tiré (en haut de chaque figure) et le touché poussé (en bas de chaque figure). Pour ces exemples particuliers la vitesse maximale de la tangente est environ de $0,65 \mathrm{~m} / \mathrm{s}\left(f a^{3}\right)$ et $0,76 \mathrm{~m} / \mathrm{s}\left(d o^{2}\right)$, comparable pour les conditions " poussé " et « tiré ». L'évolution de la vitesse de la tangente, qui oscille sous forme de sinusoïde amortie par une exponentielle, est bien visible. F0 évolue dans le temps. La mesure de $F 0$ commence environ $50 \mathrm{~ms}$ après le signal acoustique, ce qui correspond à la fenêtre temporelle nécessaire pour le calcul de ce paramètre. L'intensité au contraire est calculée instantanément sur cette figure. Pour ces deux notes, alors que les vitesses maximales sont tout à fait comparables pour les deux touchers, la F0 est plus basse 
pour le toucher tiré que pour le toucher poussé. Le toucher tiré produit un son dont l'élévation de hauteur tonale reste davantage dans une fourchette de justesse acceptable, pour une intensité sonore comparable. Il permet de jouer aussi fort en jouant plus juste.

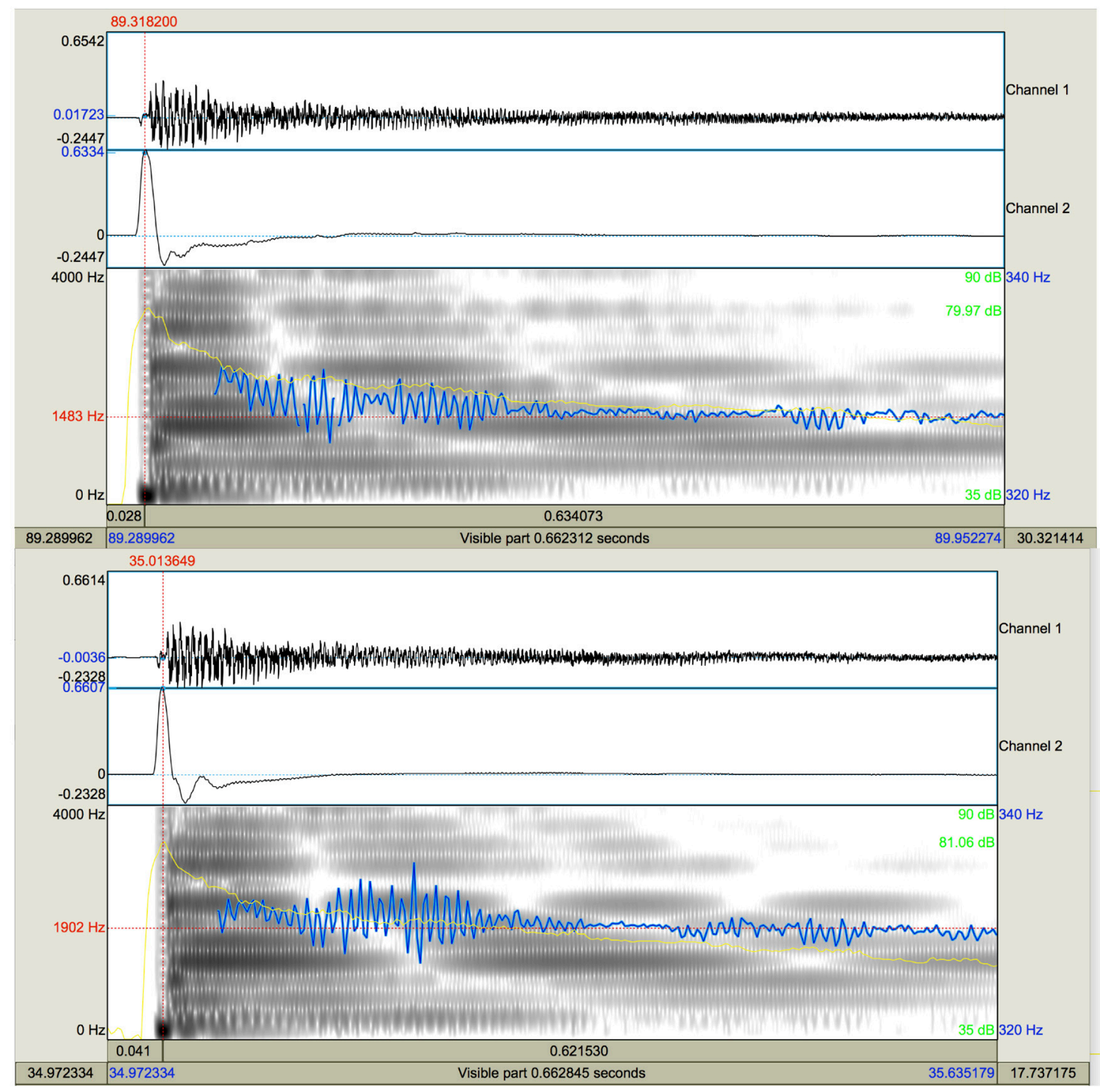

Figure $10: \mathrm{Fa}^{3}$, toucher tiré (en haut) et toucher poussé (en bas). Signal acoustique, vitesse de la tangente, spectrogramme avec la courbe d'intensité instantanée (en jaune) et la fréquence fondamentale (en bleu).

\section{LE CLAVICORDE PÉDAGOGUE}

Les mesures rapportées ici semblent confirmer l'effet de différentes techniques de jeu sur l'intonation du clavicorde, indépendamment de son intensité sonore. Le clavicorde est le seul cordophone à clavier qui permette de maintenir un contrôle 
continu de la corde par le doigt, avec des sensations tactiles accrues ${ }^{38}$. Ce contact avec la corde implique une limite fondamentale entre dynamique et intonation, puisque jouer plus fort, c'est-à-dire abaisser rapidement la touche, entraîne une plus grande élévation de la tangente donc une hausse de l'intonation. Jouer fort sans trop hausser l'intonation demande un travail particulier qui semble paradoxal : donner de la vitesse à la tangente jusqu'au moment du contact avec la corde, mais sans déplacer la corde.

Une analyse plus fine $d u$ paradoxe montre qu'un compromis entre vitesse et déplacement peut résulter du contrôle du geste dans ses différentes phases temporelles. En effet, la théorie exposée plus haut montre que le niveau sonore dépend de la vitesse d'impact de la tangente, au moment du contact (vitesse qui décroit alors très vite), donc d'un moment précis des différentes phases temporelles du toucher. L'intonation perçue dépend au contraire de la moyenne de $F 0$ sur une plage de temps plus longue, de quelques dizaines de millisecondes à une centaine de millisecondes. Durant cette plage temporelle, la tension de la corde (hauteur de la tangente) peut évoluer, et finalement donner une intonation plus basse avec une vitesse d'impact plus grande. Le toucher tiré permet donc de jouer plus juste en jouant plus fort. Cela a été remarqué de façon empirique dans une étude sur la technique du clavicorde appliquée au piano : "The playing occurs by drawing the fingers towards the player. Thus the speed (vigor) of the drawing back can determine the loudness of the tones, whereas the weight can stay constant to avoid raising the pitch » (Mühlböck 2012, p. 85).

Ce geste de retrait du doigt vers la paume de la main est à la fois très naturel (c'est le geste naturel de préhension) et très efficace pour une attaque rapide et un maintien ferme de l'abaissement de la touche tout en lui permettant de remonter légèrement : il semble donc bien que la mécanique spécifique du clavicorde soit à l'origine d'une gestuelle digitale particulière.

Le paradoxe du clavicorde semble avoir agi comme un stimulant pour développer un geste plus efficace que le geste spontanément effectué pour l'abaissement des touches. En ce sens les vertus pédagogiques reconnues du clavicorde sont objectivement reflétées par les mesures que nous proposons.

Un autre aspect des vertus pédagogiques du clavicorde est l'écoute très attentive et concentrée qu'il exige, à cause de la faiblesse relative du son. Cet aspect n'est pas considéré ici mais il est également déterminant. La sensibilité, tactile et auditive, ainsi développée semble pouvoir se transposer au jeu des autres instruments à clavier, comme le note C.P.E. Bach, aidant à la fois à l'expressivité du jeu et à la mémorisation, par renforcement entre modalités sensorielles. Le clavicorde apparaît comme un outil d'éducation somatique au clavier.

38 La question de la sensation tactile, si importante au clavicorde, se retrouvera plus tard dans différentes écoles de piano. Citons trois moments particulièrement significatifs. La méthode de piano d'Hélène de Montgeroult (ca 1788-1812), à la charnière entre XVIII ${ }^{\mathrm{e}}$ et XIX ${ }^{\mathrm{e}}$ siècles, dans la Préface, à l'article « du doigté », p. VII) décrit l'avènement de la technique nouvelle du piano-forte, en comparaison avec celle, en train de passer, du clavecin : «L'exiguïté du son vient de ce que les touches ne sont pas SENTIES mais FRAPPÉES. Les doigts, alors, sont privés d'un sens qui agit réellement sur la touche». Un siècle plus tard, c'est sans doute Marie Jaëll (Jaëll 1897), disciple de Liszt qui a poussé le plus loin son étude du toucher et l'étude des sensations tactiles au clavier. Elle ne semble pas avoir connu le clavicorde. Plus récemment, la gestuelle digitale si particulière de Glenn Gould ne manque pas d'évoquer le toucher tiré. 
D'autres écoles de clavier du XVIII e siècle que celle de Bach, par exemple les écoles issues de Jean-Philippe Rameau ou Domenico Scarlatti, demandent des techniques digitales différentes, parfois spectaculaires, et un type de virtuosité impliquant davantage le poignet ou le bras : grands intervalles, passages en octaves, acciaccatures, batteries autour du pouce, croisements rapides de main sur un même clavier. Ces différences de technique peuvent même paraître considérables. Ralph Kirkpatrick note que :

This is in direct contrast to my technique of harpsichord playing in which the back of the hand remains flexed and unchanging as a basis for the pivotal action of the fingers in overcoming the resistances of the plectra in passing the strings. In clavichord playing it remains at all times soft and malleable. In my case the difference between the two styles of playing is so great that they can hardly be recognized as emanating from the same hand (Kirkpatrick 1981, p. 305).

À la fin du XVIII ${ }^{\mathrm{e}}$ siècle, l'usage généralisé du piano et le développement d'autres techniques de virtuosité participent à l'oubli relatif du toucher tiré au profit de gestes latéraux demandant l'extension du poignet et du bras, et de la chute verticale du doigt sur le clavier, avec la participation de la main, du bras voire du corps entier.

\section{BIBLIOGRAPHIE}

Académie Française (1762), Dictionnaire de l'Académie Française, quatrième édition, Paris, Veuve Brunet.

Adlam, Derek (1998) "Clavichord Touch-Mechanics and Music », dans Bernard Brauchli, Susan Brauchli et Alberto Galazzo (dir.), De Clavicordio III, Magnano, Musica Antica a Magnano, p. 101108.

Adlung, Jakob ([1726]1768), Musica mechanica organoedi. Das ist: Gründlicher Unterricht von der Struktur, Gebrauch und Erhaltung, etc. der Orgeln, Clavicymbel, Clavichordien und anderer Instrumente, in so fern einem Organisten von solchen Sachen etwas zu wissen nöthig ist, Berlin, Birnstiel.

Alessandro, Christophe d' (2010a), "On the Dynamics of the Clavichord. From Tangent Motion to Sound " Journal of the Acoustical Society of America, vol. 128, n 4, p. 2173-2181, https://asa.scitation.org/doi/full/10.1121/1.3478783, consulté le 6 juin 2019.

Alessandro, Christophe d' (2010b) « The Acoustics of Tangent-string Interaction in the Clavichord Compared to Hammer-string Interaction in the Fortepiano ", dans Bernard Brauchli, Judith Wardman et Alberto Galazzo (dir.), De Clavicordio XI, Magnano, Musica Antica a Magnano, p. 83-90, https://groupeaa.limsi.fr/ media/membres:cda:magnano09-cda.pdf, consulté le 6 juin 2019.

Alessandro, Christophe d' (2016), «Le paradoxe du clavicorde », Actes du Congrès Français d'Acoustique, CFA 2016, Le Mans, Société Française d'Acoustique, p. 2263-2269.

Alessandro, Christophe d', Brian F.G. Katz, et François Boudet (2006), " On the Acoustics of the Clavichord ", dans Bernard Brauchli, P. Wardman et Alberto Galazzo (dir.), De Clavicordio VII, Magnano, Musica Antica a Magnano, p. 171-182.

Alessandro, Christophe d', Charles Besnainou, et Luc Ginieis (2008), « Acoustic Portrait of Four Clavichords. Tangent Velocities, Loudness and Decay Times ", dans Bernard Brauchli, Judith Wardman et Alberto Galazzo (dir.), De Clavicordio VIII, Magnano, Musica Antica a Magnano, p. 201-213.

Bach, Carl Philipp Emmanuel (1753[2009]) Versuch über die wahre Art das Clavier Zu Spielen, traduction française révisée, D. Collins, Paris, Zurfluh. 
Baffert, Jean-Marc (2018), « J.-S. Bach en France avant 1810 », Musique, images, instruments, vol. 17, Paris, Éditions du CNRS, p. 327-333.

Baillot, Etienne (1992), "Le clavicorde II. Petit mode d'emploi raisonné ", Cahiers de la Société de musique ancienne de Nice, $\mathrm{n}^{\circ} 4$, http://www.clavecin-en-france.org/spip.php?article22, consulté le 16 décembre 2018.

Bavington, Peter (1998), « Keylever, Tangent and String - A Preliminary Analysis of Clavichord Touch and Action », dans Bernard Brauchli, Susan Brauchli et Alberto Galazzo (dir.), De Clavicordio III, Magnano, Musica Antica a Magnano, p. 61-99.

Bernays, Michel, et Caroline Traube (2014), «Investigating Pianists' Individuality in the Performance of Five Timbral Nuances through Patterns of Articulation, Touch, Dynamics, and Pedaling ", Frontiers in Psychology, vol. 5, article 157 (mars), https://www.frontiersin.org/articles/10.3389/ fpsyg.2014.00157/full, consulté le 6 juin 2019.

Brauchli, Bernard (1998) The Clavichord, Cambridge, Cambridge University Press.

Brauchli, Bernard (2002) « The Clavichord as the Key to the Study of all Other Keyboard Instruments ", dans Bernard Brauchli, Alberto Galazzo et Ivan Moody (dir.), De Clavicordio V, Magnano, Musica Antica a Magnano, p. 45-62.

Cheveigné, Alain de, et Hideki Kawahara (2002) " YIN, a Fundamental Frequency Estimator for Speech and Music", Journal of the acoustical Society of America, vol. 111, no 4 (avril), p. 1917-1930, https://asa.scitation.org/doi/10.1121/1.1458024, consulté le 6 juin 2019.

Crowell, Gregory (2004), «Every Players first Grammatica », dans Bernard Brauchli, Alberto Galazzo et Ivan Moody (dir.), De Clavicordio VI, Magnano, Musica Antica a Magnano, p. 53-60.

Faulkner, Quentin (1988), "Griepenkerl on J.S. Bach's Keyboard Technique. A Translation and Commentary ", The American Organist, vol. 22, $\mathrm{n}^{\circ} 1$ (janvier), p. 63-65, https://digitalcommons. unl.edu/musicfacpub/15/, consulté le 6 juin 2019.

Ferran, Dominique (2015), "Pour un historique des doigtés anciens ", Orgue nouvelles, $\mathrm{n}^{\circ} 31$, http://www.clavecin-en-france.org/spip.php?article181, consulté le 16 décembre 2018.

Forkel, Johann Nicolaus (1802), Über Johann Sebastian Bachs Leben, Kunst und Kunstwerke, Leipzig, traduction française dans Gilles Cantagrel (1997), Bach en son temps, Paris, Fayard.

Geoffroy-Dechaume, Antoine (1986), Le langage du clavecin, Paris, Van de Velde.

Grosier, Jean-Baptiste (1780), « LETTRE XII. Essai sur la Musique ancienne \& moderne », Journal de littérature, des sciences et des arts, tome 3, p. 169-179.

Herz, Henri (1860), Méthode complète de piano, Mayence et Anvers, Schott.

Hogwood, Christopher (1988), « A Supplement to C.P.E. Bach's Versuch. E.W. Wolf's Anleitung of 1785 », dans Stephen L. Clark (dir.), C.P.E. Bach Studies, Oxford, Clarendon Press, p. 133-157.

Hülmandel, Nicolas-Joseph (1791), «Clavecin», dans Nicolas Étienne Framery et al. (dir.), Encyclopédie méthodique, $1^{\text {ère }}$ éd., tome 1, [A-Gym], Paris, Panckoucke, p. 285-286.

Jaëll, Marie (1897), Le mécanisme du toucher. L'étude du piano par l'analyse expérimentale de la sensibilité tactile, Paris, Armand Colin, https://archive.org/details/lemcanismeduto00jael/, consulté le 19 juin 2019.

Jobin, Émile (1992), «Le clavicorde I. Une voix fragile», Cahiers de la Société de musique ancienne de Nice, $\mathrm{n}^{\circ}$ 4, http://www.clavecin-en-france.org/spip.php?article21, consulté le 16 décembre 2018.

Kirkpatrick, Ralph (1981), « On Playing the Clavichord », Early Music, vol. 9, n 3 (juillet), p. 293-305, https://academic.oup.com/em/article-abstract/9/3/293/419183? redirectedFrom=PDF, consulté le 6 juin 2019.

Kooiman, Ewald (1983), " Bachs Klaviertechniek », Het Orgel, vol. 79, n 1, p. 2-15, http://www.hetorgel.nl/kooiman-ho-79-1.pdf, consulté le 6 juin 2019.

Montgeroult, Hélène de (ca 1788-1812), Cours complet pour l'enseignement du Forté Piano conduisant progressivement des premiers éléments aux plus grandes difficultés, lère partie, Paris, Launer. 
Mühlböck, Albert (2012), Recovering the Clavichord for the Modern Pianist, thèse de doctorat, Université de Cincinnati (version abrégée dans Bernard Brauchli, Judith Wardman et Alberto Galazzo (dir.), De Clavicordio XI, Magnano, Musica Antica a Magnano).

Paté, Arthur, et al. (2017), "Influence of Plectrum Shape and Jack Velocity on the Sound of the Harpsichord. An Experimental Study », Journal of the Acoustical Society of America, vol. 141, $\mathrm{n}^{\circ} 3$ (mars), p. 1523-1534, http://www.lam.jussieu.fr/Membres/LeCarrou/Articles/A17 Pate PlectrumShapeJackVelocitySoundHarpsichord.pdf, consulté le 6 juin 2019.

Quantz, Johann Joachim ([1752]1975), Essai d'une méthode pour apprendre à jouer de la flûte traversière (Versuch einer Anweisung die Flöte traversiere zu spielen), facsimilé, Paris, Zurfluh.

Speestra, Joël (2004), Bach and the Pedal Clavichord. An Organist's Guide, Rochester, University of Rochester Press.

Thwaites, Suszanne, et Neville H. Fletcher (1981), "Some Notes on the Clavichord », Journal of the Acoustical Society of America, vol. 69, $\mathrm{n}^{\circ} 5$ (mai), p. 1476-1483, https://newt.phys.unsw.edu.au/ music/people/publications/Thwaitesetal1981.pdf, consulté le 6 juin 2019.

Tournay, Jean (2009), Notes et propos sur le clavicorde, Paris, La Table Ronde.

Van Delft, Menno (2003), «Schnellen. A Quintessential Articulation Technique in Eighteenth-century Keyboard Playing », dans Christopher Hogwood (dir.), The Keyboard in Baroque Europe, Cambridge, Cambridge University Press, p. 187-97.

Walther, Johann Gotfried (1732), Musicalisches Lexicon oder Musicalische Bibliothec, Leipzig, Wolfgang Deer.

Weinreich, Gabriel (1977), "Coupled Piano Strings ", Journal of the Acoustical Society of America, vol. $62, \mathrm{n}^{\circ} 6$ (décembre), p. 1474-1485, https://asa.scitation.org/doi/10.1121/1.381677, consulté le 6 juin 2019.

Wolf, Ernst Wilhelm (1785), Eine Sonatine, Vier affectvolle Sonaten und Ein dreyzehnmal variirtes Thema, welches sich mit einer kurzen und freien Fantasie anfängt und endigst. Fürs Klavier, Leipzig, Breitkopf.

Zapf, Michael (2002), " Handing Down the Tradition. The Survival of Bach's Finger Technique in an Obscure Nineteenth-Century Clavier Tutor ", dans Bernard Brauchli, Alberto Galazzo et Ivan Moody (dir.), De Clavicordio V, Magnano, Musica Antica a Magnano, p. 39-44. 